\title{
SUI MOTI DILATATORI DI UN ELETTRONE SFERICO.
}

\author{
Memoria di Antonio Signorini (Padova).
}

Adunanza del 13 luglio 1913.

\section{IN T RODUZIONE.}

In una recente Memoria ${ }^{\mathrm{I}}$ ) ho iniziato lo studio del movimento di un elettrone in un campo elettromagnetico in base a un principio aprioristico che differisce notevolmente dagli altri fino ad oggi esperiti come punto di partenza per la meccanica dell'elettrone. Nella mia ricerca ho rinunziato ad ogni ipotesi cinematica arbitraria relativa al movimento dell'elettrone, per considerare, seguendo l'idea di Larmor, le leggi del movimento stesso come sintetizzate nell'equazione variazionale che si deduce formalmente dal principio della minima azione, quando si sostituiscano l'energia cinetica e l'energia potenziale di un sistema meccanico rispettivamente coll'energia magnetica e l'energia elettrica del campo elettromagnetico totale in cui si svolge il movimento dell'elettrone.

La differenza $L$ di queste due quantiti, quale direttamente viene fornita dall'ordinaria teoria dei fenomeni elettromagnetici, risulta una funzione della distribuzione delle cariche e delle correnti nei singoli punti dell'elettrone, e dei relativi potenziali ritardati. Non sarebbe quindi possibile esplicitare l'equazione variazionale in questione in un sistema di equazioni differenziali ordinarie se non fosse consentito:

$\mathrm{I}^{\mathrm{O}}$ considerare come finito il numero dei gradi di liberta dell'elettrone;

$2^{\circ}$ eliminare dalle espressioni dei potenziali ritardati la storia del suo movimento.

L'elettrone si differenzia da una carica elettrica generica perchè ha dimensioni infinitesime rispetto a quelle del campo in cui si svolge il suo movimento. Se si precisa questa sua proprietà qualitativa coll'ammettere che le variazioni dei parametri che definiscono l'andamento locale del movimento nei singoli suoi punti (derivate delle componenti di velocità rispetto alle coordinate) siano trascurabili di fronte ai valori dei parametri stessi in un punto qualunque, la sua deformazione più generale risulta una defor-

') A. Signorini, Sulla dinamica dell'elettrone svincolata da arbitrarie ipotesi cinematiche [Il Nuovo Cimento, serie VI, vol. IV (20 semestre 1912), pp. 257-304, 331-375]. Nel seguito indicherò questa Memoria con $(M)$. 
mazione omogenea, e corrispondentemente il numero dei suoi gradi di libertà si riduce finito (precisamente a dodici).

Con ulteriori ipotesi restrittive sull'ordine di grandezza dell'elettrone in relazione ai caratteri cinetici del suo movimento, se tale movimento si svolge con velocita inferiore alla velocità della luce, si puó, sostituite le espressioni primitive dei potenziali ritardati cogli sviluppi asintotici assegnati dal Levi-Crvita, conservare negli sviluppi stessi soltanto un numero finito di termini. Dopo ciò l'energia magnetica è l'energia elettrica del campo dell'elettrone sono ridotte a dipendere soltanto (pel tramite peró di integrali doppi di spazio) da dodici parametri e da un certo numero $v$ delle loro derivate rispetto al tempo, più o meno grande a seconda che più o meno grande è il numero dei termini che si conservano negli sviluppi asintotici. La $L$ acquista allota le proprieta formali che qualitativamente si richiedono affinchè l'equazione variazionale esprimente il principio della minima azione possa esplicitarsi in un sistema di equazioni differenziali ordinarie: cioè, secondo la denominazione introdotta da Helmholtz nel caso particolare $v={ }^{2}$ ) e successivamente generalizzata da Koenigsberger ${ }^{3}$ ), affinchè risulti il potenziale cinetico (di ordine v) del movimento dell'elettrone.

In particolare se si ammette, come ho fatto nella mia ricerca, che detti $\lambda$ ed $x$ dei limiti superiori rispettivamente della distanza di due punti dell'elettrone e della grandezza dell'accelerazione di un suo punto qualunque nel corso del suo movimento, e detta $c$ la velocità della luce nell'etere, il rapporto (puramente numerico) $\frac{\lambda x}{c^{2}}$ risulti trascurabile, gli sviluppi asintotici si possono arrestare al loro primo termine: in tale ipotesi la $L$ non dipende piu da derivate di ordine superiore al primo e viene ad avere, qualitativamente almeno, le proprietd formali di una funzione lagrangiana. Ciò ha come conseguenza immediata una notevole semplificazione dei calcoli, e questo soltanto avrebbe sommamente raccomandato di esperire in un primo studio l'ipotesi dell'infinitesimalitd del rapporto $\frac{\lambda x}{c^{2}}$, se pure non avesse quasi costretto ad ammettere l'ipotesi medesima il fatto che per essa una ricerca, che trae origine concettualmente da un principio di analogia meccanica, viene a rientrare anche per il suo sviluppo formale nello schema dell'ordinaria meccanica analitica. Invero una volta adottata tale ipotesi, se $q_{i}(i=\mathrm{r}, 2, \ldots$, I 2$)$ è un sistema di parametri corrispondenti ai singoli gradi di libertà dell'elettrone, l'equazione fondamentale si esplicita in un sistema di dodici equazioni scalari differenziali ordinarie del $2^{\circ}$ ordine, in cui il tempo funge da variabile indipendente, della forma:

$$
\frac{d}{d t} \frac{\partial L}{\partial q_{i}}-\frac{\partial L}{\partial q_{i}}=0 \quad(i=1,2, \ldots, 12),
$$

2) H. von Helmнoltz, Ueber die physikalische Bedeutung des Princips der kleinsten Wirkung [Journal für die reine und angewandte Mathematik, Bd. C (1887), pp. I37-166]; o anche: H. von Helmholtz, ITissenschaftliche Abbandlungen (Leipzig, J. A. Barth), Bd. III (I895), pp. 23 I-237.

3) L. Koenigsterger, Ueber die Principien der Mechanik [Sitzungsberichte der Kgl. Preussischen Akademie der Wissenschaften zu Berlin, Jahrgang 1896, pp. 899-944, I I73-Ir83]. 
cioè in un sistema differenziale della scconda forma di LAgrange. In generale tale sistema risulta adatto a individuate il movimento dell'elettrone quando si conosca, oltre che la specie del campo elettromagnetico in cui esso è immerso, anche la sua posizione e la distribuzione delle cariche e delle correnti elettriche ad un istante. Precisamente ciò accade tutte le volte che il sistema stesso sia risolubile rispetto alle $\ddot{q}$, ciò tutte le volte che non si annulli identicamente l'hessiano della $L$ rispetto alle $\dot{q}$.

Ricordato tutto questo, credo che non mi sarà difficile rendere chiaramente intelligibile al lettore l'origine e lo scopo del presente lavoro.

Nella Memoria $(M)$ ho iniziato lo studio del movitnento, in assenza di forze esterne, di un elettrone che ad un istante iniziale arbitrario:

$I^{\circ}$ sia sferico - colla quale denominazione ora e nel seguito intenderemo anche di esprimere che la densita elettrica è distribuita per strati sferici concentrici alla sfera che limita l'elettrone -;

$2^{\circ}$ abbia atto di movimento puramente dilatatorio - cioé la velocitì sia per tutti i suoi punti puramente radiale e proporzionale alla distanza dal centro.

Fisicamente è intuitivo che date queste condizioni iniziali e supposto nullo il campo esterno, il movimento dell'elettrone, una volta che si trascuri la storia del movimento stesso, non puó risultare altro the un movimento di pura dilatazione intorno al punto che inizialmente gli $\mathrm{fa}$ da centro: corrispondentemente a ciò in $(M)$ è dimostrato che le dodici equazioni che in generale regolano il movimento di un elettrone, quando si ammetta che il campo esterno sia nullo e l'elettrone sia sferico e dotato di movimento puramente dilatatorio, si riducono ad una sola nel parametro di dilatazione.

Studiando ulteriormente la questione ho potuto esplicitare questa equazione, ma contemporaneamente m'è saltata davanti agli occhi una circostanza da prima del tutto impreveduta : a calcoli eseguiti l'equazione non contiene più le derivate rispetto al tempo della funzione incognita, perchè lo stesso accade per la funzione lagrangiana, e non risulta soddisfatta $\mathrm{da}$ nessun valore finito del parametro di dilatazione.

Sembrerebbe dunque che la teoria da me svolta portasse necessariamente a concludere che il movimento dell'elettrone corrispondente allo stato iniziale suindicato in assenza di forze esterne, non è puramente dilatatorio: ciò che fisicamente apparisce assurdo. Ma nel fatto non è cosi, perchè a priori nulla costringe ad ammettere che tali movimenti debbano di necessita figurare tra quelli che rendono trascurabile il rapporto $\frac{\lambda \alpha}{c^{2}}$, cioè che tali movimenti debbano rientrare tra quelli effettivamente studiati in $(M)$.

Lo studio ulteriore della questione conferma pienamente che l'incongruenza da me rilevata è soltanto apparente. Volendo studiare, sempre in base alla concezione meccanica di LARmor, i movimenti di un elettrone che non sono tali da render trascurabile il rapporto $\frac{\lambda \alpha}{c^{2}}$, bisogna negli sviluppi asintotici dei potenziali ritardati conservare oltre che il primo almeno il secondo termine. Ne risulta per la $L$ un'espressione che dipende oltre che dalle derivate prime anche dalle derivate seconde dei dodici parametri 
corrispondenti ai singoli gradi di libertì dell'elettrone: cioè la $L$ viene a figurare nel problema in questione come un potenziale cinetico di $2^{\circ}$ ordine.

Esplicitando l'equazione variazionale fondamentale non si ottiene pel parametro di dilatazione dell'elettrone, come sarebbe $\mathrm{da}$ supporte a priori, un'equazione differenziale di $4^{\circ}$ ordine, ma invece un'equazione differenziale di $2^{\circ}$ ordine. Anche dopo la modificazione portata nei calcoli relativi ai moti dilatatori di un elettrone sferico, il moto di un elettrone risulta dunque sempre individuato in ogni caso dalla sua posizione e dal suo atto di movimento iniziale: cioè tale modificazione non provoca tra il modo d’individuare un moto dilatatorio di un elettrone inirialmente sferico e il modo d'individuare un generico moto di un elettrone qualunque un divario che concettualmente resterebbe di necessità ingiustificato.

Ciò depone favorevolmente all'asserto e invita allo studio dell'equazione differenziale ottenuta, che inizialmente si presenta in una forma assai complicata contenendo la derivata $\mathrm{I}^{\mathrm{a}}$ del parametro di dilatazione pel tramite di integrali doppi di spazio. Fortunatamente, se l'elettrone si suppone oltre che sferico anche omogeneo, le integrazioni possono tutte quante eseguirsi per funzioni algebriche e logaritmiche e la risoluzione dell'equazione differenziale in questione si riduce alla risoluzione di un'equazione in termini finiti seguita da una quadratura. Dopo ció facilmente si perviene alla conclusione che non si puó basare la teoria dei moti dilatatori di un elettrone sferico sull'ipotesi che il rapporto $\frac{\hat{n} \boldsymbol{x}}{c^{2}}$ sia trascurabile: invero si trova che basta che la velocità di dilatazione dell'elettrone si annulli ad un istante, o più in generale che il moto dell'elettrone sia lento, affinchè il valore di tale rapporto non risulti inferiore a $\frac{7}{9}$.

Sopprimere l'ipotesi dell'infinitesimalità del rapporto $\frac{\lambda \alpha}{c^{2}}$ non è per altro equivalente ad ammettere, come io ho fatto, che gli sviluppi asintotici dei potenziali ritardati si possano nella $L$ arrestare al $2^{\circ}$ termine. Con ciò io non ho voluto far altro che esperire nello studio dei moti dilatatori di un elettrone sferico in base alla concezione meccanica di Larmor, l'ipotesi più semplicista che era possibile ammettere.

Non dovrà dunque sorprendere, e non potrà giudicarsi in contrasto colla teoria da me svolta, il fatto che studiando l'equazione ottenuta si riconosce che essa non è atta ad individuare il movimento dell'elettrone altro che dentro limiti di tempo dipendenti dai valori iniziali del raggio dell'elettrone e della velocità di dilatazione. Tanto più che al tempo stesso si riconosce che l'equazione medesima nel suo campo di validita attribuisce ai moti dilatatori di un elettrone sferico delle proprieta che fisicamente sono perfettamente attendibili.

Riassumendo si puó dire che le conclusioni cui sono arrivato confermano pienamente l'asserto. L'incongruenza che deriva dall'applicare $i$ risultati della $(M)$ ai moti dilatatori di un elettrone sferico in assenza di forze esterne, non proviene dall'avere assunto a base della meccanica dell'elettrone la concezione di LARmor. Essa invece non è altro che una conseguenza necessaria dal fatto che le restrizioni quantitative in base alle quali è studiato il movimento dell'elettrone in $(M)$ si ripercuotono in restrizioni qua- 
litative pel movimento stesso, in quanto non sono soddisfatte da alcun movimento dilatatorio di un elettrone sferico.

\section{Capitolo I.}

\section{Generalità sul potenziale cinetico.}

I. Sia $S$ un sistema a legami completi, cioè tale che la sua posizione dipenda da un solo parametro $q$. Una funzione $L$ del tempo, di $q$ e delle derivate $\dot{q}, \ddot{q}, \ldots, q^{(v)}$ di $q$ di ordine $\leq v$ sarà a dirsi potenziale cinetico (di ordine $v$ ) del movimento del sistema $S$, tutte le volte che il movimento stesso risulti definito (col concorso della conoscenza dei valori assunti ad un istante iniziale arbitrario $t_{0}$ da $q$ e da un certo numero delle sue derivate) dall'equazione differenziale ordinaria che si ottiene esplicitando l'equazione variazionale

$$
\delta \int_{t_{1}}^{t_{2}} L d t=0
$$

ove $\left(t_{1} t_{2}\right)$ è una parte qualunque dell'intervallo di tempo in cui si considera il movimento di $S$, e $\delta$ sta a denotare una variazione hamiltoniana dell'integrale $\int_{t_{s}}^{t_{2}} L d t$ [cioè la variazione che subisce tale integrale quando dentro l'intervallo $\left(t_{1} t_{2}\right)$ si vari arbitrariamente la $q$, e insieme le $\dot{q}, \ddot{q}, \ldots, q^{(\nu)}$, lasciando inalterati $\mathrm{i}$ valori di $q, \dot{q}, \ldots, q^{(\nu-1)}$ per $t=t_{1}$, e $\left.t=t_{2}\right]$.

Siccome

$$
\delta \int_{t_{1}}^{t_{2}} L d t=\int_{t_{1}}^{t_{2}}\left(\frac{\partial L}{\partial q} \delta q+\frac{\partial L}{\partial \dot{q}} \delta \dot{q}+\frac{\partial L}{\partial \ddot{q}} \delta \ddot{q}+\cdots+\frac{\partial L}{\partial q^{(\nu)}} \partial q^{(\nu)}\right) d t
$$

$$
\delta \dot{q}=[\dot{\delta} \dot{q}] \quad \delta \ddot{q}=[\dot{\delta} \ddot{q}] \ldots \delta q^{(\nu)}=[\dot{\delta} q]^{(\nu)},
$$

si ottiene anche, mediante delle integrazioni per parti:

$$
\begin{gathered}
\delta \int_{t_{1}}^{t_{2}} L d t=\int_{t_{1}}^{t_{2}}\left(\frac{\partial L}{\partial q}-\frac{d}{d t} \frac{\partial L}{\partial \dot{q}}+\frac{d^{2}}{d t^{2}} \frac{\partial L}{\partial \ddot{q}}-\cdots+(-\mathrm{I})^{\nu} \frac{d^{\nu}}{d t^{\nu}} \frac{\partial L}{\partial q^{(\nu)}}\right) \delta q d t \\
+\left.\right|_{t_{1}} ^{t_{2}} \frac{\partial L}{\partial \dot{q}} \delta q+\left.\right|_{t_{1}} ^{t_{2}}\left(\frac{\partial L}{\partial \ddot{q}} \delta \dot{q}-\frac{d}{d t} \frac{\partial L}{\partial \ddot{q}} \delta q\right)+\cdots \\
\cdots+\left.\right|_{t_{1}} ^{t_{2}}\left(\frac{\partial L}{\partial q^{(\nu)}} \delta q^{(\nu-1)}-\frac{d}{d t} \frac{\partial L}{\partial q^{(\nu)}} \delta q^{(\nu-2)}+\cdots+(-\mathrm{I})^{\nu-1} \frac{d^{(\nu-1)}}{d t^{(\nu-1)}} \frac{\partial L}{\partial q^{(\nu)}} \delta q\right) \\
=\int_{t_{1}}^{t_{2}}\left(\frac{\partial L}{\partial q}-\frac{d}{d t} \frac{\partial L}{\partial \dot{q}}+\frac{d^{2}}{d t^{2}} \frac{\partial L}{d \ddot{q}}-\cdots+(-\mathrm{I}) \frac{d^{\nu}}{d t^{\nu}} \frac{\partial L}{\partial q^{(\nu)}}\right) \delta q d t .
\end{gathered}
$$

$\mathrm{Da}$ cio si deduce con un ragionamento ben noto che l'equazione variazionale con- 
siderata è equivalente all'equazione differenziale, in generale d'ordine $2 v$ :

$$
\frac{\partial L}{\partial q}-\frac{d}{d t} \frac{\partial L}{\partial \dot{q}}+\frac{d^{2}}{d t^{2}} \frac{\partial L}{\partial \ddot{q}}-\cdots+(-\mathrm{I})^{\nu} \frac{d^{\nu}}{d t^{\nu}} \frac{\partial L}{\partial q^{(\nu)}}=0 .
$$

2. Il potenziale cinetico dei moti dilatatori di un elettrone sferico é, come vedremo nei $\iint$ seguenti, del $2^{\circ}$ ordine e precisamente della forma

$$
L=\frac{l}{q}+\ddot{q} l^{\prime}
$$

ove $l$ ed $l$ ' sono funzioni della sola $\dot{q}$. Sembrerebbe dunque che l'equazione differenziale corrispondente dovesse risultare di $4^{\circ}$ ordine. Effettivamente però non è cosi, perchè introducendo nella (I) per $L$ la sua espressione (2) si trova semplicemente

$$
\frac{\partial}{\partial q}\left(\frac{l}{q}\right)-\frac{d}{d t} \frac{\partial}{\partial \dot{q}}\left(\frac{l}{q}\right)=0 ;
$$

cioè si trova per $q$ l'equazione differenziale del $2^{\circ}$ ordine corrispondente al potenziale cinetico ( $\mathrm{di} \mathrm{I}^{\circ}$ ordine) $\frac{l}{q}$.

E facile rendersi conto di ciò. Per ipotesi $l^{\prime}$ è una funzione della sola $\dot{q}$ : possiamo dunque considerare una funzione $L^{\prime}$ della sola $\dot{q}$ tale che sia

$$
\frac{\partial L^{\prime}}{\partial \dot{q}}=l^{\prime}
$$

Si ha allora, detti $\dot{q}_{1}$ e $\dot{q}_{2}$ i valori di $\dot{q}$ per $t=t_{\text {s }}$ e $t=t_{2}$ :

$$
\int_{t_{1}}^{t_{2}} l^{\prime} \ddot{q} d t=\int_{\dot{q}_{1}}^{\dot{q}_{2}} \frac{\partial L^{\prime}}{\partial \dot{q}} d \dot{q}=L^{\prime}\left(\dot{q}_{2}\right)-L^{\prime}\left(\dot{q}_{1}\right)
$$

e in conseguenza, poichè per $t=t_{1}$ e $t=t_{2}$ è $\delta \dot{q}=0$ :

$$
\delta \int_{i_{1}}^{t_{2}} \ddot{q} l^{\prime} d t=0
$$

ciò che prova che l'equazione variazionale, e quindi anche l'equazione differenziale, corrispondenti a un potenziale cinetico della forma

$$
\frac{l}{q}+\ddot{q} l^{\prime}
$$

devono rispettivamente coincidere con quelle corrispondenti al potenziale cinetico $\frac{l}{q}$.

La (3) puó sostituirsi colla seguente:

$$
\dot{q} \frac{\partial}{\partial q}\left(\frac{l}{q}\right)+\ddot{q} \frac{\partial}{\partial \dot{q}}\left(\frac{l}{q}\right)-\left\{\ddot{q} \frac{\partial}{\partial \dot{q}}\left(\frac{l}{q}\right)+\dot{q} \frac{d}{d t} \frac{\partial}{\partial \dot{q}}\left(\frac{l}{q}\right)\right\}=0 .
$$

Se ne deduce che è

$$
\frac{d}{d t}\left\{\frac{l}{q}-\dot{q} \frac{\partial}{\partial \dot{q}}\left(\frac{l}{q}\right)\right\}=0
$$


D'altra parte si ha identicamente

e quindi anche per la (3)

$$
\frac{l}{q}=-q \frac{\partial}{\partial q}\left(\frac{l}{q}\right)
$$

Sarà dunque

$$
\frac{l}{q}=-q \frac{d}{d t} \frac{\partial}{\partial \dot{q}}\left(\frac{l}{q}\right)
$$

$\frac{l}{q}-\dot{q} \frac{\partial}{\partial \dot{q}}\left(\frac{l}{q}\right)=-\left\{\frac{d}{d t} \frac{\partial}{\partial \dot{q}}\left(\frac{l}{q}\right)+\dot{q} \frac{\partial}{\partial \dot{q}}\left(\frac{l}{q}\right)\right\}=-\frac{d}{d t}\left\{q \frac{\partial}{\partial \dot{q}}\left(\frac{l}{q}\right)\right\}=-\frac{d}{d t} \frac{\partial l}{\partial \dot{q}}$,

$\mathrm{e}$ in conseguenza la (4) ci esprimerd che è

$$
\frac{d^{2}}{d t^{2}} \frac{\partial l}{\partial \dot{q}}=0
$$

Di qui, integrando, si deduce la relazione

$$
\frac{d}{d t} \frac{\partial l}{\partial \dot{q}}=\text { cost. }
$$

che puó, evidentemente, sostituirsi colla seguente:

$(4)^{\prime \prime}$

$$
\ddot{q} \frac{\partial^{2} l}{\partial \dot{q}^{2}}=\left[\dot{q} \frac{\partial}{\partial \dot{q}}\left(\frac{l}{q}\right)-\frac{l}{q}\right]_{i=t_{0}}
$$

Da questa, con una nuova integrazione, si ottiene

$$
\frac{\partial l}{\partial \dot{q}}=\left(\frac{\partial l}{\partial \dot{q}}\right)_{l=t_{0}}+\left[\dot{q} \frac{\partial}{\partial \dot{q}}\left(\frac{l}{q}\right)-\frac{l}{q}\right]_{t=t_{0}}\left(t-t_{0}\right)
$$

Evidentemente il primo membro della $(I)^{*}$ dipende dalla sola $\dot{q}$, il secondo soltanto da $t$ (e dai valori iniziali $q_{0}, \dot{q}_{\mathrm{o}}$ di $q$ e $\dot{q}$ ). Per essa la $q$ risulterà dunque determinata a meno della risoluzione di un'equazione in termini finiti e di una quadratura (quando siano fissati $q_{\mathrm{o}}$ e $\dot{q}_{\mathrm{o}}$ ).

3. Nella deduzione delle (4)", e $(I)^{*}$ dalla (4)' è sottinteso che la $q$ sia finita e continua insieme alla sua derivata prima.

Ammettiamo che la $l$ sia tale che non esistano valori di $\dot{q}$ per cui sia

$$
\dot{q} \frac{\partial l}{\partial \dot{q}}-l=0
$$

o almeno che $\dot{q}_{\mathrm{o}}$ sia stato scelto in modo che risulti

$$
\left(\dot{q} \frac{\partial l}{\partial \dot{q}}-l\right)_{\dot{q}=\dot{q}_{0}} \neq 0
$$

E facile provare che in queste condizioni la $\dot{q}$ non potrd mai passare per quei valori $\dot{\phi}$, eventualmente esistenti, che rendono massima o ninima la $\frac{\partial l}{\partial \dot{q}}$ considerata co- 
me funzione di $\dot{q}-\mathrm{e}$ in conseguenza, annullando la $\frac{\partial^{2} l}{\partial \dot{q}^{2}}$, rendono per la $(4)^{\prime \prime}$, infinita la $\ddot{q}$. - Infatti basta osservare che, per la $(I)^{*}, \frac{\partial l}{\partial \dot{q}}$ deve risultare una funzione lineare di $t$, cioè una funzione di $t$ sempre crescente o sempre decrescente, per concludere che non solo la $\dot{q}$ non puó riprendere due volte uno stesso valore (e quindi deve crescere o decrescere costantemente) ma anche che la $\dot{q}$ non puó prendere successivamente due valori tali che per essi coincidano i valori della $\frac{\partial l}{\partial q}$ : ciò che invece dovrebbe certamente capitare se la $\dot{q}$ passasse per un massimo o un minimo della $\frac{\partial l}{\partial \dot{q}}$.

Di più se $\dot{q}=\dot{Q}$ è effettivamente un punto di massimo (minimo) per la $\frac{\partial l}{\partial \dot{q}}$, e accade che:

$\mathrm{I}^{\circ}$ inizialmente sia $\frac{\mathrm{I}}{q}\left(\dot{q} \frac{\partial l}{\partial \dot{q}}-l\right)>0(<0)$;

$2^{\circ}$ in $\left(q_{v} \dot{Q}\right)$, escluso l'estremo $\dot{Q}$, la $\frac{\partial^{2} l}{\partial \dot{q}^{2}}$ non si annulli mai, la $q$ avrd un intervallo d'esistenza necessiriamente limitato nel futuro (nel passato) al termine (all'inizio) del quale $\dot{q}$ assume il valore $\dot{Q}$. E invero la (4)" rende evidente che se è

$$
\frac{\mathrm{I}}{q}\left(\dot{q} \frac{\partial l}{\partial \dot{q}}-l\right)>0(<0)
$$

$\ddot{q}$ e $\frac{\partial^{2} l}{\partial \dot{q}^{2}}$ avranno sempre lo stesso segno (segno opposto): in particolare che sard $\ddot{q}_{0}>0$ se $\dot{q}_{\mathrm{o}}<\dot{Q}, \ddot{q}_{\mathrm{o}}<\mathrm{o}$ se $\dot{q}_{\mathrm{o}}>\dot{Q}$. Ma $\ddot{q}$ ha costantemente lo stesso segno, perchè, come abbiamo osservato la $\dot{q}$ è sempre crescente o sempre decrescente. Accadrà dunque in ogni caso che la $\dot{q}$ dovrd variare sempre nello stesso senso approssimandosi a $\dot{Q}$. In conseguenza contemporaneamente il primo membro della $(I)^{*}$ crescerd (decrescerd) sempre, senza poter superare (divenire inferiore) al valore di $\left(\frac{\partial l}{\partial \dot{q}}\right)_{\dot{q}=\dot{Q}}$. Dopo ciò, se si osserva che il $2^{0}$ membro della $(I)^{*}$ al crescere indefinito di $t$, cresce (decresce) continuamente, l'asserto risulta evidente, in quanto l'intervallo d'esistenza della soluzione considerata non potrà estendersi nel futuro (nel passato) altro che fino a quel valore di $t$ per cui $\dot{\mathrm{e}}$

$$
t-t_{0}=\frac{\left(\frac{\partial l}{\partial \dot{q}}\right)_{\dot{q}=\dot{Q}}-\left(\frac{\partial l}{\partial \dot{q}}\right)_{\dot{q}=\dot{q}_{0}}}{\frac{\mathrm{I}}{q_{\mathrm{o}}}\left(\dot{q} \frac{\partial l}{\partial \dot{q}}-l\right)_{\dot{q}=\dot{q}_{0}}}
$$




\section{Capitolo II.}

\section{Il potenziale cinetico.}

4. Siano: $\tau$ il campo occupato da un elettrone a un istante generico $t$;

$\left(t_{1} t_{2}\right)$ una parte qualunque dell'intervallo di tempo nel quale consideriamo il suo movimento;

$\rho(Q ; t)$ e $v(Q ; t)$ la densita e la velocita di convezione dell'elettricità in un punto generico $\bar{Q}$ di $\tau$ all'istante $t$;

$\Phi_{a}(Q ; t)$ ed $A_{a}(Q ; t)$ il potenziale scalare e il potenziale vettore dell'autocampo dell'elettrone nel medesimo punto e al medesimo istante;

$c$ la velocità della luce nell'etere.

Posto

$$
L_{a}=\frac{\mathrm{I}}{2} \int_{\tau} d \tau^{\prime} \rho\left(P^{\prime} ; t\right)\left\{\frac{\nu\left(P^{\prime} ; t\right) \times A_{a}\left(P^{\prime} ; t\right)}{c}-\Phi_{a}\left(P^{\prime} ; t\right)\right\}
$$

l'equazione variazionale che secondo l'idea di LARMOR si deve assumere come sintesi delle leggi del movimento dell'elettrone in assenza di forze esterne, è la seguente

$$
\delta \int_{t_{1}}^{t_{2}} L_{a} d t=0
$$

ove il simbolo di variazione $\delta$ ha lo stesso significato che nel $\oint$ precedente, cioè sta a denotare la variazione dovuta al passaggio dal movimento effettivo dell'elettrone a un qualunque movimento variato al modo di HamiLthon ${ }^{4}$ ).

Le formole che ad un istante $t$ servono a calcolare in un punto $P^{\prime} \mathrm{i}$ valori di $\Phi_{a}$ ed $A_{a}$ quando sia conosciuta tutta quanta la storia del movimento dell'elettrone, sono ormai ben note. Indicando con $\underline{k}$ il vettore $\rho \frac{v}{c}$, tali formole sono le seguenti ${ }^{5}$ )

$$
\begin{aligned}
& \Phi_{a}\left(P^{\prime} ; t\right)=\int_{\tau} \frac{d \tau}{r} \rho\left(P ; t-\frac{r}{c}\right), \\
& A_{a}\left(P^{\prime} ; t\right)=\int_{\tau} \frac{d \tau}{r} k\left(P ; t-\frac{r}{c}\right),
\end{aligned}
$$

ove le integrazioni devono intendersi estese a tutto quanto lo spazio sopra $i$ valori assunti da $\rho$ e da $\underline{k}$ non all'istante $t$, ma all'istante che lo precede dell'intervallo di tempo $\frac{r}{c}, r$ essendo la distanza del punto generico $P$ del campo d'integrazione dal punto $P^{\prime}$.

Secondo queste formole $\Phi_{a}$ ed $A_{a}$ ci compariscono come integrali di spazio dei

4) Vedi $(M)$, pag. 2 r.

5) Cfr. ad es.: M. Aвraham, Theorie der Elektrizität (Leipzig, Teubner), Bd. II (1905), pag. 57. 
potenziali ritardati derivanti per le cariche mobili corrispondenti ai singoli punti dell'elettrone rispettivamente dalle funzioni di $t, p(t)$ e $\underline{k}(t)$. Ne segue che se sostituiamo tali potenziali ritardati coi loro sviluppi asintotici arrestati al $2^{\circ}$ termine (con che tali sviluppi vengono a dipendere soltanto dalla posizione del punto potenziato rispetto al punto potenziante e dalla velocità e dall'accelerazione del punto potenziante) per uno stesso punto $P^{\prime} \Phi_{a}$ ed $A_{a}$ si ridurranno a dipendere solamente dalla conformazione dell'elettrone e dalle velocita ed accelerazioni dei singoli suoi punti all'istante $t$.

In generale $\mathrm{i}$ primi due termini $u_{1}, u_{2}$ dello sviluppo asintotico del potenziale ritardato derivante da una funzione $\mu(t)$ per un punto potenziante (la cui velocità fino all'istante $t$ sia stata $<c$ ) sono dati da:

$$
\begin{aligned}
& u_{1}=\frac{\mu(t)}{r s}, \\
& \left.u_{2}=-\frac{\mu(t)}{2 c^{2} s^{3}}\left\{a_{r}+\beta a_{v} \beta_{r} \frac{3\left(\mathrm{I}-\beta^{2}\right)+2 \beta^{2}}{\left(\mathrm{I}-\beta^{2}\right)^{2}}\right\}-\frac{\mu(t) \beta a_{v}}{c^{2}\left(\mathrm{I}-\beta^{2}\right)^{2}}-\frac{\dot{\mu}(t)}{c\left(\mathrm{I}-\beta^{2}\right)}\left(\mathrm{I}+\frac{\beta_{r}}{s}\right){ }^{6}\right),
\end{aligned}
$$

avendo indicato :

con $r$, secondo il solito, la distanza tra il punto potenziale $P^{\prime}$ e il posto che il punto potenziante $P$ occupa all'istante $t$;

con $\beta$ e $\beta_{r}$ i quozienti per $c$ dei valori all'istante $t$ della velocita del punto potenziante $P$ e della componente di tale velocita secondo il raggio $P P^{\prime}$;

con $s$ il valore positivo del radicale $\sqrt{I-\beta^{2}+\beta_{r}^{2}}$;

con $a_{r}$ ed $a_{v}$ le componenti all'istante $t$ dell'accelerazione $a_{-}$del punto potenziante rispettivamente nel senso del moto e secondo il raggio $P P^{\prime}$.

Avremo dunque, poichè all'istante $t$ fuori di $\tau$ è $\rho=0, k=0$ :

ove

$$
\begin{aligned}
& \Phi_{a}\left(P^{\prime} ; t\right)=\Phi_{a}^{(1)}\left(P^{\prime} ; t\right)+\Phi_{a}^{(2)}\left(P^{\prime} ; t\right), \\
& \underline{A}_{a}\left(P^{\prime} ; t\right)=\underline{A}_{a}^{(1)}\left(P^{\prime} ; t\right)+\underline{A}_{a}^{(2)}\left(P^{\prime} ; t\right),
\end{aligned}
$$

$$
\Phi_{a}^{(1)}=\int_{\tau} \frac{p d \tau}{r s}, \quad A_{a}^{(s)}=\int_{\tau}^{\frac{k d \tau}{r s}},
$$

$\Phi_{a}^{(2)}=-\int_{\tau}\left[\frac{\rho}{2 c^{2} s^{3}}\left\{a_{r}+\beta a_{r} \beta_{r} \frac{3\left(\mathrm{I}-\beta^{2}\right)+2 \beta_{r}^{2}}{\left(\mathrm{I}-\beta^{2}\right)^{2}}\right\}+\frac{\rho \beta a_{v}}{c^{2}\left(\mathrm{I}-\beta^{2}\right)^{2}}+\frac{\dot{\rho}}{c\left(\mathrm{I}-\beta^{2}\right)}\left(\mathrm{I}+\frac{\beta_{r}}{s}\right)\right] d \tau$, $\underline{A}_{a}^{(2)}=-\int_{\tau}\left[\frac{k}{2 c^{2} s^{3}}\left\{a_{r}+\beta a_{\gamma^{\prime}} \beta_{r} \frac{3\left(\mathrm{I}-\beta^{2}\right)+2 \beta_{r}^{2}}{\left(\mathrm{I}-\beta^{2}\right)^{2}}\right\}+\frac{k \beta a_{v}}{c^{2}\left(\mathrm{I}-\beta^{2}\right)^{2}}+\frac{\dot{k}}{c\left(\mathrm{I}-\beta^{2}\right)}\left(\mathrm{I}+\frac{\beta_{r}}{s}\right)\right] d \tau$.

Sostituendo queste espressioni di $\Phi_{a}$ ed $A_{a}$ nella $(5)$ si ottiene subito

$$
L_{a}=L_{a}^{(1)}+L_{a}^{(2)}
$$

6) Vedi: T. Levi-Crvira, Sulla espressione asintotica dei potenziali ritardati [Atti del IV ${ }^{\circ}$ Congresso Internazionale dei Matematici (Roma, 6-I I aprile 1908), vol. III (1909), pp. 89-100]. 
ove

(6)

$$
\begin{aligned}
& L_{a}^{(\mathrm{I})}=\frac{\mathrm{I}}{2} \int_{\tau} \frac{\mid v^{\prime} \times A_{a}^{(1 \mathrm{I})}}{c}-\frac{\left.\Phi_{a}^{\prime(1)}\right\}}{c} \rho^{\prime} d \tau^{\prime}=\frac{\mathrm{I}}{2} \int_{\tau} \frac{\rho \rho^{\prime}}{r s}\left(\frac{v^{\prime} \times \underline{v}}{c^{2}}-\mathrm{I}\right) d \tau d \tau^{\prime}, \\
& L_{a}^{(2)}=\frac{\mathrm{I}}{2} \int_{\tau}\left\{\frac{v^{\prime} \times A_{a}^{\prime(2)}}{c}-\Phi_{a}^{\prime(2)}\right\} \rho^{\prime} d \tau^{\prime} \\
& \left.\left.=-\frac{\mathrm{I}}{2 c^{2}} \int_{\tau} \rho \rho^{\prime}\left(\frac{v^{\prime} \times \underline{v}}{c^{2}}-\mathrm{I}\right)\left[\frac{\mathrm{I}}{2 s^{3}}\right\}_{r}+\beta a_{v} \beta_{r} \frac{3\left(\mathrm{I}-\beta^{2}\right)+2 \beta_{r}^{2}}{\left(\mathrm{I}-\beta^{2}\right)^{2}}\right\}+\frac{\rho \beta a_{v}}{\left(\mathrm{I}-\beta^{2}\right)^{2}}\right] d \tau d \tau^{\prime} \\
& -\frac{\mathrm{I}}{2 c} \int_{\tau} \frac{\rho^{\prime}}{\mathrm{I}-\beta^{2}}\left(\frac{\underline{v}^{\prime} \times \dot{k}}{c}-\dot{\rho}\right)\left(\mathrm{I}+\frac{\beta_{r}}{s}\right) d \tau d \tau^{\prime} \text {. }
\end{aligned}
$$

5. Per lo studio dei movimenti (in assenza di forze esterne) di un elettrone inizialmente sferico e dotato di atto di movimento puramente dilatatorio, potremo fin da ora supporre la $L_{a}$ calcolata in base all'ipotesi che il movimento dell'elettrone sia puramente dilatatorio: perchè tale dovendo risultare in conseguenza delle supposte condizioni iniziali, la $(I)$ si riduce ad esprimere che è nullo l'incremento che assume l'integrale $\int_{t_{1}}^{t_{2}} L_{a} d t$ quando nell'intervallo $\left(t_{1} t_{2}\right)$ si varì al modo di HamiLton il parametro di dilatazione dell'elettrone.

Siano allora :

$\tau_{0}$ il.campo (sferico) inizialmente occupato dall'elettrone;

$P_{\mathrm{o}}, P_{\mathrm{o}}^{\prime}$ le posizioni iniziali di $P, \because$;

$r_{\mathrm{o}}$ la distanza di $P_{\mathrm{o}}$ e $P_{\mathrm{o}}^{\prime}$;

$B_{\mathrm{o}}$ ed $A$ rispettivamente il centro ed il raggio della sfera $\tau_{0}$;

$\underline{\alpha}, \underline{\alpha^{\prime}}$ i vettori $B_{\mathrm{o}} P_{\mathrm{o}}, B_{\mathrm{o}} P_{\mathrm{o}}^{\prime}$;

$\theta$ l'angolo di tali vettori;

$\gamma$ l'angolo dei raggi $P_{\mathrm{o}} B_{\mathrm{o}}$ e $P_{\mathrm{o}} P_{\mathrm{o}}^{\prime}$;

$\psi$ il valore all'istante $t$ del parametro di dilatazione dell'elettrone, cioè il valore del quoziente per $A$ del raggio dell'elettrone a tale istante; con che per $t=t_{\mathrm{o}}$ risulterd $\psi=\mathrm{r}$.

Per il fatto che il movimento dell'elettrone è puramente dilatatorio evidentemente sarà:

$$
\begin{array}{lll}
r=\psi r_{0}, & \underline{v}=\ddot{\psi} \alpha, \quad \beta= \pm \frac{\dot{\psi} \alpha}{c}, & \beta_{r}=-\frac{\dot{\psi} \alpha \cos \gamma}{c}, \\
s=\sqrt{\mathrm{I}-\frac{\alpha^{2} \dot{\psi}^{2}}{c^{2}} \sin ^{2} \gamma}, & \underline{a}=\ddot{\psi} \alpha, \quad a_{v}= \pm \ddot{\psi} \alpha, \quad a_{r}=-\ddot{\psi} \alpha \cos \gamma,
\end{array}
$$

ove sard da scegliere il segno $+0-$ secondochè è $\psi>0$ oppure $<0$.

Se inoltre indichiamo con $p_{\mathrm{o}}$ il valore all'istante $t_{\mathrm{o}}$ della densità elettrica in $P_{\mathrm{o}}$ [valore che per l'ipotesi della sfericità dell'elettrone potrà dipendere soltanto da $x$ ] e con $d \tau_{\mathrm{o}}$ l'elemento di $\tau_{\mathrm{o}}$ circostante a $P_{\mathrm{o}}$ che rappresenta la posizione iniziale dell'elemento $d \tau$ di $\tau$ circostante a $P$, come conseguenza immediata del principio di conservazione 
dell'elettricità avremo ${ }^{7}$ ):

$$
\rho_{0} d \tau_{0}=\rho d \tau \text {. }
$$

Di più, le derivate di $f(t)$ e $k(t)$ che compariscono nella $(6)_{2}$ dovendo intendersi eseguite seguendo il movimento delle singole cariche elementari corrispondenti ai punti dell'elettrone, sempre pel principio di conservazione dell'elettricità avremo:

cioè

$$
\dot{p}+p \operatorname{div} \underline{v}=0
$$

$$
\dot{p}=-\frac{3 \dot{\psi} p}{\psi}
$$

$\mathrm{e}$

$$
\dot{k}=-\frac{3 \dot{\psi}^{2} p \alpha}{c \dot{\psi}}+\frac{p \ddot{\psi} \underline{\alpha}}{c}
$$

$\mathrm{Ne}$ risulta in definitiva che il potenziale cinetico dei moti dilatatori di un elettrone sferico sard dato da

ove si assuma

$$
L_{a}=L_{a}^{(1)}+l_{a}^{(2)}+\ddot{\psi} l_{a}^{(2)}
$$

$$
\left\{\begin{array}{c}
L_{1}^{(1)}=\frac{I}{2 \psi} \int_{\tau_{0}} \rho_{0} \rho_{0}^{\prime} \frac{\frac{\dot{\psi}^{2} \alpha \alpha^{\prime} \cos \theta}{c^{2}}-\mathrm{I}}{r_{\mathrm{o}} \sqrt{\mathrm{I}-\frac{\dot{\psi}^{2} \alpha^{2}}{c^{2}} \sin ^{2} \gamma} d \tau_{0} d \tau_{0}^{\prime},} \\
l_{a}^{(2)}=\frac{3 \dot{\psi}}{2 c \psi} \int_{\tau_{0}} \rho_{0} \rho_{0}^{\prime}\left(\mathrm{I}-\frac{\frac{\alpha \dot{\psi}}{c} \cos \gamma}{\sqrt{\mathrm{I}-\frac{\dot{\psi}^{2} \alpha^{2}}{c} \sin ^{2} \gamma}}\right) \frac{\frac{\dot{\psi}^{2} \alpha \alpha^{\prime} \cos \theta}{c^{2}}-\mathrm{I}}{\mathrm{I}-\frac{\alpha^{2} \dot{\psi}^{2}}{c^{2}}} d \tau_{0} d \tau_{0}^{\prime}
\end{array}\right.
$$

e si indichi con $l_{a}^{(2)}$ una funzione della sola $\dot{\psi}$. Gia a questo punto è provato che il potenziale cinetico dei moti dilatatori di un elettrone sferico possiede le proprietá formali che per esso abbiamo ammesse al Cap. I. Da ciò segue in primo luogo che l'equazione differenziale che ne deriva è un'equazione di $2^{\circ}$ ordine, non del $4^{\circ}$ come si potrebbe supporre a priori per il fatto che $L_{a}$ dipende da $\ddot{\psi}$ : onde la soluzione di tale equazione corrispondente a un determinato moto dilatatorio di un elettrone sferico, risulterd individuata appena siano noti (oltre che la specie della $p_{0}$ ) il raggio e l'atto di movimento iniziale dell'elettrone. Di più dalle conclusioni del Cap. I segue che per il calcolo di tale soluzione è superflua la conoscenza dell'espressione effettiva di $l_{a}^{\prime(2)}$ in funzione di $\dot{\psi}$ : una volta nota l'espressione effettiva di $\psi\left(L_{a}^{(1)}+l_{a}^{(2)}\right)$ in funzione di $\psi$, il calcolo della soluzione medesima porrd eseguirsi in base alla $(I)^{*}$ e si ridurrd alla risoluzione di un'equazione in termini finiti per la $\psi$, e ad una quadratura. 
Nel seguito porremo per semplicità per un generico valore di $s$

e in particolare

$$
\frac{s \psi}{c}=q_{s}, \quad \frac{s \dot{\psi}}{c}=\dot{q}
$$

$$
\frac{A \psi}{c}=q, \quad \frac{A \dot{\psi}}{c}=\dot{q} .
$$

Con ciò le (7) assumono la forma più semplice

$$
\begin{aligned}
L_{a}^{(\mathrm{I})} & =\frac{A}{2 c q} \int_{\tau_{0}} \frac{\dot{q}_{a} \dot{q}_{\alpha^{\prime}} \cos \theta-\mathrm{I}}{r_{0} \sqrt{\mathrm{I}-\dot{q}_{\alpha}^{2} \sin ^{2} \gamma}} \rho_{0} \rho_{0}^{\prime} d \tau_{0} d \tau_{0}^{\prime}, \\
l_{a}^{(2)} & =\frac{3 \dot{q}}{2 c q} \int_{\tau_{0}} \frac{\dot{q}_{\alpha} \dot{q}_{a^{\prime}} \cos \theta-\mathrm{I}}{\mathrm{I}-\dot{q}_{x}^{2}}\left(\mathrm{I}-\frac{\dot{q}_{a} \cos \gamma}{\sqrt{\mathrm{I}-\dot{q}_{\alpha}^{2} \sin ^{2} \gamma}}\right) \rho_{0} \rho_{0}^{\prime} d \tau_{0} d \tau_{0}^{\prime} .
\end{aligned}
$$

Si noti che dalla nostra ipotesi

segue direttamente

$$
\begin{gathered}
v<c \\
|\dot{q}|<\mathrm{I} .
\end{gathered}
$$

\section{Capitolo III.}

\section{Calcolo del $\mathrm{I}^{\circ}$ termine del potenziale cinetico.}

6. Nel capitolo precedente abbiamo stabilito che il contributo portato nel potenziale cinetico dei moti dilatatori di un elettrone sferico dal primo termine degli sviluppi asintotici dei potenziali ritardati - cioè (vedi Introduzione) la funzione lagrangiana dei moti stessi - è dato da

$$
L_{a}^{(1)}=\frac{A}{2 c q} \int_{\tau_{0}} \rho_{0} \rho_{0}^{\prime} d \tau_{0} d \tau_{0}^{\prime} \frac{\mathrm{I}}{r_{0}} \frac{\dot{q}_{\alpha} \dot{q}_{\alpha^{\prime}} \cos \theta-\mathrm{I}}{\sqrt{\mathrm{I}-\dot{q}_{\alpha}^{2} \sin ^{2} \gamma}} .
$$

E evidente che per la sfericità dell'elettrone l'integrale

$$
\int_{\tau_{0}} \rho_{0}^{\prime} d \tau_{0}^{\prime} \frac{I}{r_{0}} \frac{\dot{q}_{\alpha} \dot{q}_{\alpha^{\prime}} \cos \theta-\mathrm{I}}{\sqrt{\mathrm{I}-\dot{q}_{\alpha}^{2} \sin ^{2} \gamma}} .
$$

dipende da $P_{\mathrm{o}}$ soltanto pel tramite della sua distanza $\propto$ da $B_{0}$. Da questa osservazione segue immediatamente che $\mathrm{e}$

$$
L_{a}^{(1)}=\frac{2 \pi A}{c q} \int_{0}^{A} \rho_{0} \alpha^{2} d \alpha \int_{\tau_{0}} \rho_{0}^{\prime} d \tau_{0}^{\prime} \frac{I}{r_{0}} \frac{\dot{q}_{\alpha} \dot{q}_{x^{\prime}} \cos \theta-\mathrm{I}}{\sqrt{\mathrm{I}-\dot{q}_{\alpha}^{2} \sin ^{2} \gamma}} .
$$

Se poi supponiamo lo spazio riferito a un sistema di coordinate polari $\left(x^{\prime}, \theta, \varphi\right)$ avente per origine il punto $B_{\mathrm{o}}$ e per asse polare il raggio $B_{0} P_{0}$, la funzione

$$
\frac{\rho_{0}^{\prime}}{r_{0}} \frac{\dot{q}_{a} \dot{q}_{a^{\prime}} \cos \theta-\mathrm{I}}{\sqrt{\mathrm{I}-\dot{q}_{a}^{2} \sin ^{2} \gamma}}
$$


risulterd indipendente dalla longitudine $\rho$ del punto $P^{\prime}$ e, poichè

sarà

$$
d \tau^{\prime}=\alpha^{\prime 2} \sin \theta d \alpha^{\prime} d \theta d \varphi
$$

$$
L_{a}^{(1)}=\frac{4 \pi^{2} A}{c q} \int_{0}^{d} \rho_{0} \alpha^{2} d x \int_{0}^{A} \rho_{0}^{\prime} \alpha^{\prime 2} d \alpha^{\prime} \int_{0}^{\pi} \frac{\sin \theta d \theta}{r_{0}} \frac{\dot{q}_{\alpha} \dot{q}_{\alpha^{\prime}} \cos \theta-\mathrm{I}}{\sqrt{\mathrm{I}-\dot{q}_{\alpha}^{2} \sin ^{2} \gamma}}
$$

pur d'intendere che attualmente il punto $P^{\prime}$ cui si riferiscono $r_{\mathrm{o}}$ e $\gamma$ appartenga sempre ad una stessa tra le infinite semicirconferenze sezioni della sfera di centro $B_{\circ}$ e raggio $\alpha^{\prime}$, con un semipiano limitato al raggio $B_{0} P_{0}$, e quindi sia perfettamente individuato dal valore di $\theta$ ad esso relativo.

7. Indichiamo con $i$ l'integrale (che evidentemente non puó dipendere altro che da $\alpha$ ed $x^{\prime}$ )

dimostreremo che si ha:

$$
\int_{0}^{\pi} \frac{\sin \theta d \theta}{r_{0}} \frac{\dot{q}_{x} \dot{q}_{x^{\prime}} \cos \theta-\mathrm{I}}{\sqrt{\mathrm{I}-\dot{q}_{x}^{2} \sin ^{2} \gamma}} .
$$

$$
\left\{\begin{array}{l}
i=-\frac{2}{\alpha} \quad \text { per } \quad \alpha^{\prime} \leq x, \\
i=-\frac{2}{\alpha^{\prime}} \text { per } \quad \alpha^{\prime} \supseteq \alpha .
\end{array}\right.
$$

Ciò porta come immediata conseguenza

$$
L_{a}^{(\mathrm{I})}=-\frac{8 \pi^{2} A}{c q} \int_{0}^{A} p_{0} \alpha d \alpha\left\{\int_{0}^{\alpha} \alpha^{\prime 2} p_{0}^{\prime} d \alpha^{\prime}+\alpha \int_{\alpha}^{A} \alpha^{\prime} p_{0}^{\prime} d \alpha^{\prime}\right\}
$$

e prova che, come ho accennato nell'Introduzione, la funzione lagrangiana dei moti dilatatori di un elettrone sferico risulta indipendente dalla velocita di dilatazione.

Poniamo

$\mathrm{e}$ in conseguenza

$$
\alpha^{\prime}=e \alpha
$$

$$
\dot{q}_{\alpha^{\prime}}=e \dot{q}_{\alpha} \text {. }
$$

Riferendosi alla definizione degli angoli $\theta$ e $\gamma$ si vede subito che $\dot{e}$

$$
\begin{gathered}
r_{0} \sin \gamma=\alpha^{\prime} \sin \theta=e \alpha \sin \theta, \\
r_{0}^{2}=\alpha^{2}+\alpha^{\prime 2}-2 \alpha \alpha^{\prime} \cos \theta=\alpha^{2}\left(1+e^{2}-2 e \cos \theta\right) .
\end{gathered}
$$

$\mathrm{Ne}$ risulta per $i$ la seguente espressione:

$$
i=\frac{\mathrm{I}}{\alpha} \int_{0}^{\pi} \frac{\left(e \dot{q}_{\alpha}^{2} \cos \theta-\mathrm{I}\right) \sin \theta d \theta}{\sqrt{\mathrm{I}+e^{2}-2 e \cos \theta}-e^{2} \dot{q}_{\alpha}^{2} \sin ^{2} \theta} .
$$

Cambiamo in questo integrale la variabile d'integrazione assumendo come tale

Sard allora

$$
x=\cos \theta \text {. }
$$

$$
\begin{aligned}
i & =\frac{\mathrm{I}}{\alpha} \int_{-1}^{1} \frac{\left(e \dot{q}_{a}^{2} x-\mathrm{I}\right) d x}{\sqrt{e^{2} \dot{q}_{\alpha}^{2} x^{2}-2 e x+\mathrm{I}+e^{2}\left(\mathrm{I}-\dot{q}_{\alpha}^{2}\right)}} \\
& =\frac{\mathrm{I}}{\alpha e} \int_{-1}^{\mathrm{I}} d \sqrt{e^{2} q_{\alpha}^{2} x^{2}-2 e x+\mathrm{I}+e^{2}\left(\mathrm{I}-\dot{q}_{\alpha}^{2}\right)} \\
& =\frac{\mathrm{I}}{\alpha e}\{ \pm(\mathrm{I}-e)-(\mathrm{I}+e)\},
\end{aligned}
$$


ove è da scegliere il segno $+o$ il segno - secondochè è

oppure

$e \leq \mathrm{I}$

cioé secondochè è

oppure

Nella prima ipotesi si trova

$$
e \gg \mathrm{I}
$$

$$
\alpha^{\prime} \leq \alpha
$$$$
\alpha^{\prime} \supseteq \alpha
$$

nella seconda

$$
i=-\frac{2 e}{\alpha e}=-\frac{2}{\alpha}
$$

$$
i=-\frac{2}{\alpha e}=-\frac{2}{\alpha^{\prime}} .
$$

Con questo le (8) e insieme la (9) restano dimostrate e in conseguenza resta pure dimostrato che la funzione lagrangiana dei moti dilatatori di un elettrone sferico è indipendente dalla velocità di dilatazione ${ }^{8}$ ).

8. Se si suppone l'elettrone oltre che sferico anche omogeneo, le integrazioni che compariscono nel secondo membro della (9) si eseguiscono a vista e si ottiene, indicando con $p_{o}$ il valore iniziale comune a tutti $i$ punti dell'elettrone:

$$
L_{a}^{(\mathrm{r})}=-\frac{\mathrm{I} 6}{\mathrm{I} 5} \pi^{2} \frac{\rho_{0}^{2} A^{6}}{q c}
$$

Vedremo nel capitolo seguente che in tale ipotesi puó eseguirsi in termini finiti anche il calcolo di $l_{a}^{(2)}$.

\section{Capitolo IV.}

\section{Calcolo del secondo termine (semplificato) del potenziale cinetico.}

9. Per il calcolo di $l_{a}^{(2)}$ nell'ipotesi che l'elettrone sia omogeneo, è essenziale la determinazione dell'integrale

$$
i_{l}=\int_{\tau_{0}} d \tau_{\mathrm{o}}^{\prime}\left(\mathrm{I}-\frac{\dot{q}_{\alpha} \cos \gamma}{\sqrt{\mathrm{I}-\dot{q}_{\alpha}^{2} \sin ^{2} \gamma}}\right)\left(\dot{q}_{\alpha} \dot{q}_{\alpha^{\prime}} \cos \theta-\mathrm{I}\right)
$$

in quanto in tale ipotesi si ha evidentemente

$l_{a}^{(2)}=\frac{3}{2} \frac{\dot{q}}{q c} \int_{\tau_{0}} \frac{p_{0} \rho_{0}^{\prime} d \tau_{0} d \tau_{0}^{\prime}}{\mathrm{I}-\dot{q}_{\alpha}^{2}}\left(\mathrm{I}-\frac{\dot{q}_{\alpha} \cos \gamma}{\sqrt{\mathrm{I}-\dot{q}_{\alpha}^{2} \sin ^{2} \gamma}}\right)\left(\dot{q}_{\alpha} \dot{q}_{\alpha^{\prime}} \cos \theta-\mathrm{I}\right)=6 \pi \frac{\dot{q} \rho_{0}^{2}}{q c} \int_{0}^{A} \frac{\alpha^{2} d \alpha i_{l}}{\mathrm{I}-\dot{q}_{\alpha}^{2}}$.

8) Ė facile vedere che dal risultato ora ottenuto segue che l'hessiano della funzione lagrangiana del piủ generale movimento dell'elettrone rispetto alle $\dot{q}$ (vedi Introduzione) risulta identicamente nullo per un elettrone sferico se si suppone il suo movimento quasi dilatatorio [Cfr. $(M), \S 22$ ]. Per un elettrone inizialmente sferico non è dunque accettabile l'ipotesi di semplificazione formale della funzione lagrangiana introdotta a scopo puramente euristico nell'ultimo capitolo della mia Memoria ( $M$ ). 
Immaginando associati nell'integrale

$$
\int_{\tau_{\mathrm{o}}} \dot{q}_{\alpha} \dot{q}_{\alpha^{\prime}} \cos \theta d \tau_{\mathrm{o}}^{\prime}
$$

gli elementi corrispondenti a punti simmetrici rispetto al piano diametrale di $\tau_{0}$ perpendicolare al raggio $P_{o} B_{o}$, si riconosce subito che tale integrale è identicamente nullo. Onde, se si pone

sarà

$$
\begin{aligned}
i_{1}^{(1)} & =\int_{\tau_{0}} \frac{\dot{q}_{\alpha} \cos \gamma}{\sqrt{I-\dot{q}_{\alpha}^{2} \sin ^{2} \gamma}} d \tau_{0}^{\prime}=\dot{q}_{\alpha} \int_{\tau_{0}} \frac{\cos \gamma d \tau_{0}^{\prime}}{\sqrt{\prime}-\overline{\dot{q}_{\alpha}^{2} \sin ^{2} \gamma}}, \\
i_{l}^{(2)} & =\int_{\tau_{0}} \frac{\dot{q}_{\alpha} \cos \gamma \cdot \dot{q}_{\alpha} \dot{q}_{\alpha^{\prime}} \cos \theta}{\sqrt{\mathrm{I}-\dot{q}_{\alpha}^{2} \sin ^{2} \gamma}} d \tau_{0}^{\prime}=\dot{q}_{\alpha}^{2} \int \frac{\dot{q}_{x} \cos \gamma \cos \theta d \tau_{0}^{\prime}}{\sqrt{\mathrm{I}-\dot{q}_{\alpha}^{2} \sin ^{2} \gamma}},
\end{aligned}
$$

perchè è

$$
i_{l}=-\frac{4}{3} \pi A^{i}+i_{l}^{(\mathrm{r})}-i_{l}^{(2)},
$$

$$
\int_{\tau_{0}} d \tau_{0}^{\prime}=\frac{4}{3} \pi A^{3}
$$

Corrispondentemente, essendo

$$
\int_{0}^{A} \frac{\alpha^{2} d \alpha}{\mathrm{I}-\dot{q}_{\alpha}^{2}}=\frac{A^{2}}{\dot{q}^{2}}\left\{-A+\int_{0}^{A} \frac{d \alpha}{\mathrm{I}-\dot{q}_{\alpha}^{2}}\right\}=\frac{A^{2}}{\dot{q}^{2}}\left\{-A+\frac{A}{2 \dot{q}} \log \frac{\mathrm{I}+\dot{q}}{\mathrm{I}-\dot{q}}\right\},
$$

se poniamo

avremo

$$
I_{1}^{(1)}=\frac{6 \pi \dot{q} \dot{p}_{0}^{2}}{q c} \int_{0}^{A} \frac{x^{2} d x i_{l}^{(1)}}{\mathrm{I}-\dot{q}_{x}^{2}}, \quad I_{1}^{(2)}=\frac{6 \pi \dot{q} \rho_{0}^{2}}{q c} \int_{0}^{A} \frac{x^{2} d x i_{l}^{(2)}}{\mathrm{I}-\dot{q}_{x}^{2}},
$$

$$
l_{a}^{(2)}=\frac{8 \pi^{2} A^{6} \rho_{0}^{2}}{q \dot{q} c} l_{I}-\frac{\mathrm{I}}{2 \dot{q}} \log \frac{\mathrm{I}+\dot{q})}{\mathrm{I}-\dot{q})}+I_{l}^{(1)}-I_{l}^{(2)} \text {. }
$$

ro. Supponiamo lo spazio riferito a un sistema di coordinate polari $\left(r_{0}, \gamma, v\right)$ avente per origine il punto $P_{\mathrm{o}}$ e per asse polare il raggio $P_{\mathrm{o}} B_{\mathrm{o}}$. Si ha allora

e la funzione

$$
d \tau_{\mathrm{o}}^{\prime}=r_{\mathrm{o}}^{2} \sin \gamma d r_{\mathrm{o}} d \gamma d v
$$

$$
\frac{\cos \gamma}{\sqrt{I-\dot{q}_{x}^{2} \sin ^{2} \gamma}}
$$

risulta indipendente dalla longitudine $v$ del punto $P^{\prime}$. Sarà dunque

$$
i_{l}^{(1)}=2 \pi \dot{q}_{\alpha} \int_{0}^{\pi} \frac{\sin \gamma \cos \gamma d \gamma}{\sqrt{\mathrm{I}-\dot{q}_{\alpha}^{2} \sin ^{2} \gamma}} \int_{0}^{R_{\gamma}} r_{0}^{2} d r_{0}=\frac{2}{3} \pi \dot{q}_{x} \int_{0}^{\pi} \frac{R_{\gamma}^{3} \cos \gamma \sin \gamma d \gamma}{\sqrt{\mathrm{I}-\dot{q}_{\alpha}^{2} \sin ^{2} \gamma}},
$$

ove si indichi con $R_{\Upsilon}$ la distanza da $P_{o}$ (contata in valore assoluto) di uno qualunque dei punti di $\tau_{0}$ corrispondenti al valore $\gamma$ della colatitudine. Evidentemente $R_{\curlyvee}$ ed $R_{\pi-\Upsilon}$ ci dànno le due radici dell'equazione di secondo grado

$$
\xi^{2}-2 \alpha \cos \gamma \xi+\alpha^{2}-A^{2}=0
$$


onde risulterà

$$
\left.R_{\gamma}^{3}-R_{\pi_{-\gamma}}^{j}=8 \alpha^{3} \cos ^{3} \gamma-6 \alpha \cos \gamma\left(\alpha^{2}-A^{2}\right)^{9}\right) .
$$

In conseguenza, quando nell'integrale che compare nell'espressione ora ottenuta per $i_{l}^{(\Sigma)}$ si associno gli elementi corrispondenti a valori supplementari di $\gamma$, avremo

$$
\begin{gathered}
i_{l}^{(1)}=\frac{2}{3} \pi \dot{q}_{\alpha} \int_{0}^{\frac{\pi}{2}}\left\{8 \alpha^{3} \cos ^{3} \gamma-6 x \cos \gamma\left(x^{2}-A^{2}\right)\right\} \frac{\sin \gamma \cos \gamma d \gamma}{\sqrt{\mathrm{I}-\dot{q}_{\alpha}^{2} \sin ^{2} \gamma}} \\
=\frac{\mathrm{I} 6}{3} \pi \alpha^{3} \dot{q}_{\alpha} \int_{0}^{\frac{\pi}{2}} \frac{\cos ^{4} \gamma \sin \gamma d \gamma}{\sqrt{\mathrm{I}-\dot{q}_{\alpha}^{2} \sin ^{2} \gamma}}-4 \pi x\left(x^{2}-A^{2}\right) \dot{q}_{\alpha} \int_{0}^{\frac{\pi}{2}} \frac{\cos ^{2} \gamma \sin \gamma d \gamma}{\sqrt{\mathrm{I}-\dot{q}_{\alpha}^{2} \sin ^{2} \gamma}}
\end{gathered}
$$

Ora qualunque sia $m$ (purchè intero $\mathrm{e} \gg \mathrm{o}$ ) l'integrale

$$
I_{m}(p)=\int_{0}^{\frac{\pi}{2}} \frac{\cos ^{2 m} \gamma \sin \gamma d \gamma}{\sqrt{I-p^{2} \sin ^{2} \gamma}}
$$

ove $p$ è una costante minore dell'unità, si esprime per funzioni algebriche e logaritmiche di $p$.

Infatti, sostituendo in $I_{m}(p)$ la variabile $\gamma$ colla variabile $z$ definita dalla relazione

si trova subito

$$
\frac{I+\frac{p^{2}}{I-p^{2}} \cos ^{2} \gamma}{\cos ^{2} \gamma}=z^{2}
$$

$$
I_{m}(p)=\frac{\mathrm{I}}{\sqrt{\mathrm{I}-p^{2}}} \int_{\frac{1}{\sqrt{I-p^{2}}}}^{\infty} \frac{d z}{\left(z^{2}-\frac{p^{2}}{\mathrm{I}-p^{2}}\right)^{m+1}}=\left.\frac{\mathrm{I}}{\pi(m) \sqrt{I-p^{2}}}\right|_{\frac{1}{\sqrt{I-p^{2}}}} ^{\infty}\left[\frac{d^{m}}{d c^{m}} \int \frac{d z}{z^{2}-c}\right]_{c=-\frac{p^{2}}{\mathrm{I}-p^{2}}}
$$

e infine, poichè

$$
\begin{aligned}
& \int \frac{d z}{z^{2}-c}=\frac{1}{2 \sqrt{c}} \log \frac{z-\sqrt{c}}{z+\sqrt{c}}+\text { cost., } \\
& \left\{I_{m}(p)=\frac{1}{\pi(m) \sqrt{\mathrm{I}-\bar{p}^{2}}}\left\{\left(\frac{d^{m}}{d c^{m}}\right\}_{\frac{\mathrm{I}}{2 \sqrt{c}}} \log \frac{z-\sqrt{c})}{z+\sqrt{c}}\right\}\right)_{c=\frac{p^{2}}{i-p^{2}}, z=\infty} \\
& -\left(\frac{d^{m}}{d c^{m}}\left\{\frac{\mathrm{I}}{2 \sqrt{c}} \log \frac{z-\sqrt{c})}{z+\sqrt{c})}\right)\right\}_{c=\frac{p^{2}}{1-p^{2}}, i=\frac{1}{\sqrt{1-p^{2}}}}
\end{aligned}
$$

9) Il metodo seguito nel testo per la determinazione di $i_{l}^{(1)}$ e successivamente di $i_{l}^{(2)}$ è stato da me adoperato anche in $(M)$ pel calcolo di alcuni integrali [Cfr. $(M), \S 23]$.

In seguito ho potuto riconoscere che esso puó servire anche a ritrovare la formola di MAcLAURIN relativa al potenziale di un elissoide omogeneo in un punto interno, e allora soltanto, consultando $\mathrm{i}$ lavori che si occupano di teoria del potenziale, ho appreso che esso era già stato adoperato a tale scopo da Lord Kelvin [Cfr.: Lord Kelvin and P. G. TAIT, A Treatise on Natural Philosophy (Cambridge, University Press), Part II (1895), pag. 44].

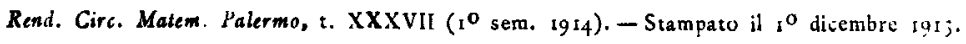


Se ne deduce che in particolare sard

$$
\left\{\begin{array}{l}
\left.\left.\int_{0}^{\frac{\pi}{2}} \frac{\cos ^{2} \gamma \sin \gamma d \gamma}{\sqrt{\mathrm{I}-\dot{q}_{\alpha}^{2} \sin ^{2} \gamma}}=I_{1}\left(\dot{q}_{x}\right)=\frac{\mathrm{I}}{2 \dot{q}_{\alpha}^{2}}\right\}_{\mathrm{I}}-\frac{\mathrm{I}}{2 \dot{q}_{x}}\left(\mathrm{I}-\dot{q}_{x}^{2}\right) \log \frac{\mathrm{I}+\dot{q}_{x}}{\mathrm{I}-\dot{q}_{x}}\right\}, \\
\left.\int_{0}^{\frac{\pi}{2}} \frac{\cos ^{4} \gamma \sin \gamma d \gamma}{\sqrt{\mathrm{I}-\dot{q}_{\alpha}^{2} \sin ^{2} \gamma}}=I_{2}\left(\dot{q}_{\alpha}\right)=\frac{\mathrm{I}}{8 \dot{q}_{\alpha}^{4}} \int^{5} \dot{q}_{\alpha}^{2}-3+\frac{3}{2 \dot{q}_{\alpha}}\left(\mathrm{I}-\dot{q}_{\alpha}^{2}\right)^{2} \log \frac{\left.\mathrm{I}+\dot{q}_{\alpha}\right)}{\mathrm{I}-\dot{q}_{\alpha}}\right)
\end{array}\right.
$$

Dopo ciò si perviene senza difficoltà alla seguente espressione di $i_{l}^{(1)}$ :

$i_{l}^{(\mathrm{I})}=\pi A^{j}\left\{-\frac{2}{\dot{q}^{3}}+\frac{2}{\dot{q}}+\frac{4}{3} \frac{\alpha^{2}}{A^{2} \dot{q}}+\frac{A}{\alpha \dot{q}^{2}}\left(\mathrm{I}-\dot{q}_{\alpha}^{2}\right)\left(\frac{\mathrm{I}}{\dot{q}^{2}}-\mathrm{I}\right) \log \frac{\left.\mathrm{I}+\dot{q}_{\alpha}\right\}}{\mathrm{I}-\dot{q}_{\alpha}}\right.$.

Ne segue

$$
\left.I_{l}^{(\mathrm{I})}=\frac{\mathrm{I} 2 \pi^{2} \rho_{0}^{2} A^{3}}{q c} \int_{0}^{A} \frac{\alpha^{2} d \alpha}{\mathrm{I}-\dot{q}_{\alpha}^{2} !}-\frac{\mathrm{I}}{\dot{q}^{2}}+\mathrm{I}+\frac{2}{3} \frac{\alpha^{2}}{A^{2}}\right)+\frac{6 \pi^{2} \rho_{0}^{2} \dot{A}^{4}}{\dot{q} q c}\left(\frac{\mathrm{I}}{\dot{q}^{2}}-\mathrm{I}\right) \int_{0}^{A} \log \frac{\mathrm{I}+\dot{q}_{\alpha} \alpha d \alpha}{\mathrm{I}-\dot{q}_{\alpha}}
$$

ed anche, integrando per parti il $2^{\circ}$ integrale del $2^{\circ}$ membro (assumendo in esso per fattor differenziale $\alpha d \alpha$ :

$$
\begin{aligned}
& I_{l}^{(1)}=\frac{3 \pi^{2} \dot{\rho}_{0}^{2} A^{6}}{\dot{q} q c}\left(\frac{\mathrm{I}}{\dot{q}^{2}}-\mathrm{I}\right) \log \frac{\mathrm{I}+\dot{q}}{\mathrm{I}-\dot{q}}+\frac{\mathrm{I} 8 \pi^{2} \rho_{0}^{2} A^{3}}{q c} \int_{0}^{A} \frac{\alpha^{2} d x}{\mathrm{I}-\dot{q}_{\alpha}^{2}}\left(\mathrm{I}-\frac{\mathrm{I}}{\dot{q}^{2}}+\frac{4}{9} \frac{\alpha^{2}}{A^{2}}\right) \\
& =\frac{3 \pi^{2} p_{0}^{2} A^{6}}{\dot{q} q c}\left(\frac{\mathrm{I}}{\dot{q}^{2}}-\mathrm{I}\right) \log \frac{\mathrm{I}+\dot{q}}{\mathrm{I}-\dot{q}}+\frac{\mathrm{I} 8 \pi^{2} \rho_{0}^{2} A^{3}}{q c} \int_{0}^{A}\left\{-\frac{4}{3} \frac{\alpha^{2}}{\dot{q}^{2}}-\frac{A^{2}}{\dot{q}^{2}}\left(\mathrm{I}-\frac{5}{9 \dot{q}^{2}}\right)\right\} d \alpha \\
& +\frac{18 \pi^{2} p_{0}^{2} A^{5}}{\dot{q}^{2} q c}\left(\mathrm{I}-\frac{5}{q \dot{q}^{2}}\right) \int_{0}^{A} \frac{d \alpha}{\mathrm{I}-\bar{q}_{x}^{2}} \\
& =\frac{3 \pi^{2} p_{0}^{2} A^{6}}{\dot{q} q c}\left(\frac{\mathrm{I}}{\dot{q}^{2}}-\mathrm{I}\right) \log \frac{\mathrm{I}+\dot{q}}{\mathrm{I}-\dot{q}}-\frac{8}{3} \frac{\pi^{2} \rho_{0}^{2} A^{6}}{\dot{q}^{2} q c}-\frac{\mathrm{I} 8 \pi^{2} p_{0}^{2} A^{6}}{\dot{q}^{2} q c}\left(\mathrm{I}-\frac{5}{9 \dot{q}^{2}}\right) \\
& +\frac{q \pi^{2} \rho_{0}^{2} A^{6}}{\dot{q}^{3} q c}\left(\mathrm{I}-\frac{5}{9 \dot{q}^{2}}\right) \log \frac{\mathrm{I}+\dot{q}}{\mathrm{I}-q}
\end{aligned}
$$

ed infine

$$
\left\{\begin{array}{l}
I_{l}^{(1)}=-\frac{62}{3} \frac{\pi^{2} p_{0}^{2} A^{6}}{\dot{q}^{2} q c}+\frac{\mathrm{IO} \pi^{2} p_{0}^{2} A^{6}}{\dot{q}^{4} q c}+\frac{\pi^{2} \rho_{0}^{2} A^{6}}{\dot{q} q c} \log \frac{\mathrm{I}+\dot{q}}{\mathrm{I}-\dot{q}}\left\{-3+\frac{\mathrm{I} 2}{\dot{q}^{2}}-\frac{5}{\dot{q}^{4}}\right\} \\
\left.\left.=\frac{\pi^{2} p_{0}^{2} A^{6}}{q c}\right\}-\frac{62}{3 \dot{q}^{2}}+\frac{\mathrm{IO}}{\dot{q}^{4}}+\frac{\mathrm{I}}{\dot{q}} \log \frac{\mathrm{I}+\dot{q}}{\mathrm{r}-\dot{q}}\left\{-3+\frac{\mathrm{I} 2}{\dot{q}^{2}}-\frac{5}{\dot{q}^{4}}\right\}\right\} .
\end{array}\right.
$$

A riprova dell'esattezza dei calcoli si puó constatare che sostituendo per $\log \frac{\mathrm{I}+\dot{q}}{\mathrm{I}-\dot{q}}$ il suo sviluppo in serie di potenze di $\dot{q}$, si ottiene

$$
I_{l}^{(\mathrm{r})}=\frac{4^{8}}{35} \frac{\pi^{2} \rho_{\mathrm{o}}^{2} A^{6}}{q c} \dot{q}^{2}\{\mathrm{I}+\cdots\}
$$

ove gli infiniti termini omessi dentro la parentesi contengono ognuno a fattore una potenza di $\dot{q}$ (non inferiore alla $2^{\mathbf{a}}$ ). Cio da un lato prova che la forma d'infinito che 
prende l'espressione trovata per $I_{l}^{(1)}$ quando si ponga $\dot{q}=0$, è soltanto apparente: e d'altro lato, sempre per $\dot{q}=o$, fornisce come valore del limite del rapporto del valore di tale espressione a $q^{2}$, lo stesso valore che si ottiene calcolando direttamente per $\dot{q}=0$ il quoziente $\frac{I_{l}^{1)}}{\dot{q}^{2}}$.

I I. Il calcolo di $I_{l}^{(2)}$ si compie in modo del tutto analogo. Dalla definizione degli angoli $\theta$ e $\gamma$ risulta immediatamente che è

$$
\dot{q}_{\alpha^{\prime}} \cos \theta=\dot{q}_{\alpha}-\frac{r_{0} \dot{q}}{A} \cos \gamma .
$$

D'altra parte dalla (12) segue che è

$$
R_{\gamma}^{4}+R_{\pi-\gamma}^{4}=16 \alpha^{4} \cos ^{4} \gamma-16\left(x^{2}-A^{2}\right) x^{2} \cos ^{2} \gamma+2\left(\alpha^{2}-A^{2}\right)^{2} .
$$

Avremo dunque successivamente

$$
\begin{aligned}
& i_{l}^{(2)}=\dot{q}_{\alpha}^{2} \int_{\tau_{0}} \frac{\dot{q}_{\alpha^{\prime}} \cos \gamma \cos \theta d \tau_{0}^{\prime}}{\sqrt{\mathrm{I}-\dot{q}_{\alpha}^{2} \sin ^{2} \gamma}}=\dot{q}_{\alpha}^{2} \int_{\tau_{0}} \frac{\cos \gamma\left(\dot{q}_{\alpha}-\frac{r_{0} \dot{q}}{A} \cos \gamma\right)}{\sqrt{\mathrm{I}-\dot{q}_{\alpha}^{2} \sin ^{2} \gamma}} d \tau_{0}^{\prime} \\
& =2 \pi \dot{q}_{\alpha}^{3} \int_{0}^{\pi} \frac{\sin \gamma \cos \gamma d \gamma}{\sqrt{\mathrm{I}-\dot{q}_{\alpha}^{2} \sin ^{2} \gamma}} \int_{0}^{R_{\gamma}} r_{0}^{2} d r_{0}-2 \pi \frac{\dot{q} \dot{q}_{\alpha}^{2}}{A} \int_{0}^{\pi} \frac{\sin \gamma \cos ^{2} \gamma d \gamma}{\sqrt{\mathrm{I}-\dot{q}_{\alpha}^{2} \sin ^{2} \gamma}} \int_{0}^{R_{\gamma}} r_{0}^{3} d r_{0} \\
& =\frac{2}{3} \pi \dot{q}_{\alpha}^{3} \int_{0}^{\frac{\pi}{2}} \frac{\sin \gamma \cos \gamma d \gamma}{\sqrt{\mathrm{I}-\dot{q}_{\alpha}^{2} \sin ^{2} \gamma}}\left\{R_{\gamma}^{3}-R_{\pi-\gamma}^{;}\right\}-\frac{\mathrm{I}}{2} \pi \frac{\dot{q} \dot{q}_{\alpha}^{2}}{A} \int_{0}^{\frac{\pi}{2}} \frac{\sin \gamma \cos ^{2} \gamma d \gamma}{\sqrt{\mathrm{I}-\dot{q}_{\alpha}^{2} \sin ^{2} \gamma}}\left\{R_{\gamma}^{4}+R_{\pi-r}^{4}\right\} \\
& =\frac{4}{3} \pi x \dot{q} \int_{0}^{\frac{\pi}{2}} \frac{\sin \gamma \cos ^{2} \gamma d \gamma}{\sqrt{\mathrm{I}-\dot{q}_{\alpha}^{2} \sin ^{2} \gamma}}\left\{4 x^{2} \cos ^{2} \gamma-3\left(\alpha^{2}-A^{2}\right)\right\} \\
& -\frac{\pi \dot{q} \dot{q}_{\alpha}^{2}}{A} \int_{0}^{\frac{\pi}{2}} \frac{\sin \gamma \cos ^{2} \gamma d \gamma}{\sqrt{1-\dot{q}_{\alpha}^{2} \sin ^{2} \gamma}}\left\{8 \alpha^{4} \cos ^{4} \gamma-8 \alpha^{2}\left(\alpha^{2}-A^{2}\right) \cos ^{2} \gamma+\left(\alpha^{2}-A^{2}\right)^{2}\right\} \\
& =-\pi\left(s \alpha^{3} \dot{q}_{x}^{3}-6 \alpha A^{2} \dot{q}_{\alpha}^{3}+A^{3} \dot{q} \dot{q}_{\alpha}^{2}\right) \int_{0}^{\frac{\pi}{2}} \frac{\sin \gamma \cos ^{2} \gamma d \gamma}{\sqrt{\mathrm{I}-\dot{q}_{\alpha}^{2} \sin ^{2} \gamma}} \\
& +\pi\left(\frac{40}{3} \alpha^{3} \dot{q}_{\alpha}^{3}-8 \alpha A^{2} \dot{q}_{\alpha}^{j}\right) \int_{0}^{\frac{\pi}{2}} \frac{\sin \gamma \cos ^{+} \gamma d \gamma}{\sqrt{\mathrm{I}-\dot{q}_{\alpha}^{2} \sin ^{2} \gamma}}-8 \pi \alpha^{3} \dot{q}_{\alpha}^{3} \int_{0}^{\frac{\pi}{2}} \frac{\sin \gamma \cos ^{6} \gamma d \gamma}{\sqrt{\mathrm{I}-\dot{q}_{\alpha}^{2} \sin ^{2} \gamma}} \text {. }
\end{aligned}
$$

Dalla (1 3 ) si deduce senza difficoltà

$$
\int_{0}^{\frac{\pi}{2}} \frac{\sin \gamma \cos ^{6} d \gamma}{\sqrt{\mathrm{I}-\dot{q}_{\alpha}^{2} \sin ^{2} \gamma}}=I_{3}(\dot{q})=\frac{\mathrm{I}}{4^{8} \dot{q}_{\alpha}^{6}}\left\{33 \dot{q}_{\alpha}^{4}-40 \dot{q}_{\alpha}^{2}+15-\frac{\mathrm{I} 5}{2 \dot{q}_{\alpha}}\left(\mathrm{I}-\dot{q}_{\alpha}^{2}\right)^{3} \log \frac{\mathrm{I}+\dot{q}_{\alpha}}{\mathrm{I}-\dot{\dot{q}_{\alpha}}}\right\} .
$$

Tenendo conto di ció e delle (I4) si perviene in definitiva alla seguente espressione di $i_{l}^{(2)}$ :

$$
i_{l}^{(2)}=\pi A^{3}\left\{\frac{\alpha^{3}}{3 A^{3}} \dot{q}_{\alpha}-\frac{2 \alpha}{A} \dot{q}_{\alpha}-\frac{\dot{q}}{2}+\frac{5 x^{2}}{3 \dot{q} A^{2}}+\frac{3}{\dot{q}}-\frac{5}{2 \dot{q}^{3}}+\frac{A}{\alpha}\left(\mathrm{I}-\dot{q}_{\alpha}^{2}\right)\left(\frac{\mathrm{I}}{4}-\frac{3}{2 \dot{q}^{2}}+\frac{5}{4 \dot{q}^{4}}\right)\right\} .
$$


Ne segue

$$
\begin{aligned}
& \left.I_{l}^{(2)}=\frac{6 \pi^{2} p_{0}^{2} \dot{q} A^{3}}{q c} \int_{0}^{A} \frac{\alpha^{2} d \alpha}{\mathrm{I}-\dot{q}_{\alpha}^{2}} \frac{\alpha^{3}}{\left(3 A^{3}\right.} \dot{q}_{\alpha}-\frac{2 \alpha}{A} q_{\alpha}-\frac{\dot{q}}{2}+\frac{5 x^{2}}{3 \dot{q} A^{2}}+\frac{3}{\dot{q}}-\frac{5}{2 \dot{q}^{3}}\right) \\
& +\frac{3 \pi^{2} p_{0}^{2} \dot{q} A^{4}}{q c}\left\{\frac{1}{2}-\frac{3}{q^{2}}+\frac{5}{\left.4 \dot{q}^{4}\right)} \int_{0}^{A} \log \frac{\mathrm{I}+\dot{q}_{x}}{\mathrm{I}-\dot{q}_{x}} x d \alpha\right. \\
& =\frac{3}{2} \frac{\pi^{2} \rho_{0}^{2} \dot{q} A^{6}}{q c}\left\{\frac{\mathrm{I}}{2}-\frac{3}{\dot{q}^{2}}+\frac{5}{4 \dot{q}^{4}}\right\} \log \frac{\mathrm{I}+\dot{q}}{\mathrm{I}+\dot{q}} \\
& +\frac{3 \pi^{2} \rho_{0}^{2} A}{q c} \int_{0}^{A} d \alpha\left\{-\frac{2}{3} x^{4}+4\left[\mathrm{I}-\frac{\mathrm{I}}{\dot{q}^{2}}\right] A^{2} \alpha^{2}+\left[\frac{7}{2 \dot{q}^{4}}-\frac{5}{\dot{q}^{2}}+\frac{3}{2}\right] A^{4}\right\} \\
& +\frac{3 \pi^{2} \rho_{0}^{2} A^{5}}{q c}\left\{-\frac{7}{2 \dot{q}^{4}}+\frac{5}{\dot{q}^{2}}-\frac{3}{2}\right\} \int_{0}^{A} \frac{d \alpha}{\mathrm{I}-\dot{q}_{x}^{2}} \\
& =\frac{3}{2} \frac{\pi^{2} \rho_{0}^{2} \dot{q}}{q c} A^{6}\left\{\frac{\mathrm{I}}{2}-\frac{3}{\dot{q}^{2}}+\frac{5}{2 \dot{q}^{4}}\right\} \log \frac{\mathrm{I}+\dot{q}}{\mathrm{I}-\dot{q}}+\frac{3 \pi^{2} \rho_{0}^{2} A^{6}}{q c}\left\{\frac{8 \mathrm{I}}{\mathrm{IO}}-\frac{\mathrm{I} 9}{3 \dot{q}^{2}}+\frac{7}{2 \dot{q}^{4}}\right\} \\
& \left.+\frac{3 \pi^{2} p_{0}^{2} A^{6}}{\dot{q} q c}-\frac{7}{2 \dot{q}^{4}}+\frac{5}{\dot{q}^{2}}-\frac{3}{2}\right\} \log \frac{1+\dot{q}}{1-\dot{q}} \\
& \left.=\frac{8 \mathrm{I}}{\mathrm{IO}} \frac{\pi^{2} p_{0}^{2} A^{6}}{q c}-\frac{\mathrm{I} 9 \pi^{2} p_{0}^{2} A^{6}}{\dot{q}^{2} q c}+\frac{2 \mathrm{I}}{2} \frac{\pi^{2} p_{0}^{2} A^{6}}{\dot{q}^{4} q c}+\frac{\pi^{2} \rho_{0}^{2} A^{6}}{q c} \frac{13}{(4} q-\frac{27}{4 \dot{q}}+\frac{45}{4 \dot{q}^{3}}-\frac{2 \mathrm{I}}{4 \dot{q}^{5}}\right) \log \frac{\mathrm{I}+\dot{q}}{\mathrm{I}-\dot{q}}
\end{aligned}
$$

e infine

(I6) $I_{l}^{(2)}=\frac{\pi^{2} \rho_{0}^{2} A^{6}}{q \mathcal{C}}\left\{\frac{8 \mathrm{I}}{10}-\frac{\mathrm{I} 9}{\dot{q}^{2}}+\frac{2 \mathrm{I}}{2 \dot{q}^{4}}+\frac{3}{2} \dot{q}^{5}(\mathrm{I}-\dot{q})^{2}\left(\frac{\mathrm{I}}{2} \dot{q}^{4}+\dot{q}^{3}-3 \dot{q}^{2}-7 \dot{q}-7\right)^{\prime}\right.$

Anche qui si può avere una riprova dell'esattezza dei calcoli constatando che quando si sostituisca a $\log \frac{\mathrm{I}+\dot{q}}{\mathrm{I}-\dot{q}}$ il suo sviluppo in serie di potenze di $\dot{q}$ si ottiene

$$
I_{i}^{(2)}=-\frac{4^{8}}{315} \frac{\pi^{2} p_{0}^{2} A^{6}}{q c} \dot{q}^{4}\{1+\cdots\}
$$

(ove gli infiniti termın omessi tra parentesi contengono ognuno a fattore una potenza di $q$ non inferiore alla seconda) e insieme che il calcolo diretto del rapporto $\frac{I_{l}^{(2)}}{\dot{q}^{4}}$ per $q=0, \mathrm{da}$ il risultato

$$
\left(\frac{I_{l}^{(2)}}{\dot{q}^{4}}\right)_{\dot{q}=0}=-\frac{4^{8}}{3 I 5} \frac{\pi^{2} p_{0}^{2} A^{10}}{q c^{5}}
$$

Tenendo conto delle (I5), (I6), la (II) ci dad immediatamente

$$
\begin{aligned}
l_{l}^{(2)} & \left.=-\frac{\pi^{2} p_{0}^{2} A^{6}}{q c} \frac{8 \mathrm{I}}{(\mathrm{IO}}-\frac{8}{\dot{q}}+\frac{5}{3 \dot{q}^{2}}+\frac{\mathrm{I}}{2 \dot{q}^{4}}-\log \frac{\mathrm{I}+\dot{q}}{\mathrm{I}-\dot{q}}\left(-\frac{3}{4} \dot{q}+\frac{\mathrm{I} 5}{4 \dot{q}}-\frac{4}{\dot{q}^{2}}+\frac{3}{4 \dot{q}^{3}}+\frac{\mathrm{I}}{4 \dot{q}^{5}}\right)\right\} \\
& =-\frac{\pi^{2} p_{0}^{2} A^{6}}{q c}\left\{\frac{81}{\mathrm{I} 0}-\frac{8}{\dot{q}}+\frac{5}{3 \dot{q}^{3}}+\frac{\mathrm{I}}{2 \dot{q}^{4}}-\frac{\mathrm{I}}{4 \dot{q}^{5}}(\mathrm{I}-\dot{q})^{3}\left(3 \dot{q}^{3}+9 \dot{q}^{2}+3 \dot{q}+\mathrm{I}\right) \log \frac{\mathrm{I}+q}{\mathrm{I}-\dot{q})}\right.
\end{aligned}
$$




\section{Capitolo V.}

\section{L'equazione dei moti dilatatori di un elettrone sferico omogeneo. Conseguenze.}

12. I risultati ottenuti negli ultimi due capitoli si possono riassumere col dire che il potenziale cinetico dei moti dilatatori di un elettrone sferico omogeneo è della forma

$$
L_{a}=\frac{l_{a}}{q}+\ddot{q} l_{a}^{\prime},
$$

ove $l_{a}$ ed $l_{a}^{\prime}$ sono funzioni della sola $\dot{q}$, e precisamente è

$$
\text { (I7) }\left\{\begin{array}{c}
l_{a}=q\left(L_{a}^{(\mathrm{I})}+l_{a}^{(2)}\right) \\
=-\frac{\pi^{2} \rho_{0}^{2} A^{6}}{c}\left\{\begin{array}{c}
55 \\
6
\end{array}-\frac{8}{\dot{q}}+\frac{5}{3 \dot{q}^{2}}+\frac{\mathrm{I}}{2 \dot{q}^{4}}-\frac{\mathrm{I}}{4 \dot{q}_{5}}(\mathrm{I}-\dot{q})^{3}\left(3 \dot{q}^{3}+q \dot{q}^{2}+3 \dot{q}+\mathrm{I}\right) \log \frac{\mathrm{I}+\dot{q}}{\mathrm{I}-\dot{q}}\right\} \\
=-\frac{\pi^{2} \rho_{0}^{2} A^{6}}{c}\left\{\frac{\mathrm{I} 6}{\mathrm{I} 5}+\frac{8}{3} \dot{q}-\frac{48}{35} \dot{q}^{2}+\frac{8}{5} \dot{q}^{3}+\cdots\right\},
\end{array}\right.
$$

gli infiniti termini omessi tra parentesi contenendo ognuno a fattore una potenza di $\dot{q}$ non inferiore alla $L_{a}$.

E subito visto che $\frac{\partial l_{q}}{\partial \dot{q}}$ quando $\dot{q}$ varia da o ad I passa almeno per un massimo.

Infatti è evidentemente

mentre, essendo

$$
\left(\frac{\partial^{2} l_{a}}{\partial \dot{q}^{2}}\right)_{\dot{q}=0}=\frac{96}{35} \frac{\pi^{2} \rho_{0}^{2} A^{6}}{c}>0,
$$

si ha

$$
\lim _{\dot{q}=1}\left\{\frac{\partial^{2}}{\partial \dot{q}^{2}}(\mathrm{I}-\dot{q})^{3} \log \frac{\mathrm{I}+\dot{q}}{\mathrm{I}-\dot{q}}\right\}=0
$$

$$
\left(\frac{\partial^{2} l_{a}}{\partial \dot{q}^{2}}\right)_{\dot{q}=1}=-4 \frac{\pi^{2} \rho_{o}^{2} A^{6}}{c}<0
$$

Rileveremo di più che è

$$
\left(\dot{q} \frac{\partial l}{\partial \dot{q}}-l\right)_{\dot{q}=0}=\frac{16}{15} \frac{\pi^{2} p^{2} A^{6}}{c}>0 .
$$

13. Sostituiamo ad $l$ nella $(I)^{*}$ l'espressione cui siamo pervenuti per $l_{a}$. Otterremo cosi un'equazione in termini finiti tra $\dot{q}$ e $t$ della forma seguente :

$$
\left\{\begin{array}{c}
\frac{8}{\dot{q}^{2}}-\frac{10}{3 \dot{q}^{3}}-\frac{2}{\dot{q}^{5}}-\frac{1}{2 \dot{q}^{5}} \frac{(1-\dot{q})^{2}}{\mathrm{I}+\dot{q}}\left(3 \dot{q}^{3}+9 \dot{q}^{2}+3 \dot{q}+1\right) \\
+\frac{3 \dot{q}^{4}+6 \dot{q}^{3}+24 \dot{q}^{2}+10 \dot{q}+5}{4 \dot{q}^{6}} \log \frac{\mathrm{I}+\dot{q}}{\mathrm{I}-\dot{q}}\left(\mathrm{I}-\dot{q}_{0}\right)^{2}=c_{0}+c_{0}^{\prime} t
\end{array}\right.
$$


ove $c_{\mathrm{o}}$ e $c_{\mathrm{o}}^{\prime}$ dipendono soltanto da $\dot{q}_{\mathrm{o}}$ e precisamente sono definite ponendo:

$$
\begin{aligned}
& c_{0}=\frac{8}{\dot{q}_{0}^{2}}-\frac{\mathrm{IO}}{3 \dot{q}_{0}^{3}}-\frac{2}{\dot{q}_{0}^{5}}-\frac{\mathrm{I}}{2 \dot{q}_{0}^{5}} \frac{\left(\mathrm{I}-\dot{q}_{0}\right)^{2}}{\mathrm{I}+\dot{q}_{0}}\left(3 \dot{q}_{0}^{3}+9 \dot{q}_{0}^{2}+3 \dot{q}_{0}+\mathrm{I}\right) \\
& +\frac{\left(\mathrm{I}-\dot{q}_{0}\right)^{2}}{4 \dot{q}_{0}^{6}}\left(3 \dot{q}_{0}^{+}+6 \dot{q}_{0}^{3}+10 \dot{q}_{0}^{2}+10 \dot{q}_{0}+5\right) \log \frac{\mathrm{I}+q_{0}}{\mathrm{I}-\dot{q}_{0}}, \\
& c_{\mathrm{o}}^{\prime}=\frac{c}{A}\left\{-\frac{55}{6}+\frac{\mathrm{I} 6}{\dot{q}_{\mathrm{o}}}-\frac{5}{\dot{q}_{\mathrm{o}}^{2}}-\frac{5}{2 \dot{q}_{\mathrm{o}}^{4}}-\frac{\mathrm{I}}{2 \dot{q}_{\mathrm{o}}^{4}} \frac{\left(\mathrm{I}-\dot{q}_{\mathrm{o}}\right)^{2}}{\mathrm{I}+\dot{q}_{\mathrm{o}}}\left(3 \dot{q}_{\mathrm{o}}^{3}+9 \dot{q}_{\mathrm{o}}^{2}+3 \dot{q}_{\mathrm{o}}+\mathrm{I}\right)\right. \\
& \left.+\frac{3\left(5 \dot{q}_{0}^{2}+2 \dot{q}_{0}+1\right)}{2 \dot{q}_{0}^{5}}\left(\mathrm{I}-\dot{q}_{0}\right)^{2} \log \frac{\mathrm{I}+\dot{q}_{0}}{\mathrm{I}-\dot{q}_{\mathrm{o}}}\right\} \text {. }
\end{aligned}
$$

Questa è l'equazione che in base alle conclusioni del Cap. I - supposto, s'intende, assegnato il valore iniziale $\dot{q}_{0}$ di $q_{0}$ - individuerà il movimento dell'elettrone per tutto l'intervallo di tempo in cui ammetterà una soluzione che soddisfi alla condizione iniziale $(\dot{q})_{l=t_{0}}=\dot{q}_{\circ}$. Per essa il calcolo effettivo della $q$, che equivale perfettamente a quello del parametro di dilatazione dell'elettrone, sard ricondotto alla risoluzione di un'equazione in termini finiti e ad una quadratura.

Sempre in base alle conclusioni del Cap. I, risulta evidente che, almeno nell'intervallo di tempo nel quale il movimento dell'elettrone resta determinato dalla $(I)$, se il movimento stesso ad un istante sard accelerato (ritardato) sarà costantemente accelerato (ritardato); di più (essendosi rilevato che la $\frac{\partial l}{\partial \dot{q}}$ quando $q$ varia da o ad i passa almeno per un massimo, e che per $q=0$ è: $q \frac{\partial l}{\partial \dot{q}}-l>0$ ) si riconosce che l'equazione ottenuta non è atta ad individuare qualunque moto dilatatorio di un elettrone sferico omogeneo in qualunque intervallo di tempo.

14. Sviluppando il primo membro della $(I)$ in serie di potenze di $\dot{q}$ e $c_{0}, c_{0}^{\prime}$ in serie di potenze di $q_{0}$, la $(I)$ assume la forma

$\frac{8}{3}-\frac{96}{35} \dot{q}+\frac{24}{5} \dot{q}^{2}+\cdots=\frac{8}{3}-\frac{96}{35} \dot{q}_{0}+\frac{24}{5} \dot{q}_{0}^{2}+\cdots+\frac{c}{A}\left\{-\frac{16}{15}-\frac{48}{35} \dot{q}_{0}^{2}+\frac{16}{5} \dot{q}_{0}^{3}+\cdots\right\}\left(t-t_{0}\right)$.

Derivandola rispetto a $t$ avremo dunque

$$
\ddot{q}\left\{\frac{96}{35}+\cdots\right\}=\frac{c}{A}\left\{\frac{16}{15}+\cdots\right\}
$$

ove gli infiniti termini omessi dentro le parentesi contengono ognuno a fattore una potenza di $\dot{q}$ o $\dot{q}_{0}$. Ne segue senz'altro che se ad un istante il valore di $\dot{q}$ (cioè quello della velocita di dilatazione $\dot{\psi}$ ) sarà nullo, allo stesso istante avremo, conservando naturalmente il simbolo $A$ a denotare il raggio dell'elettrone all'istante medesimo,

$$
\ddot{q}=\frac{A \ddot{\psi}}{c}=\frac{c}{A} \cdot \frac{16}{15} \cdot \frac{35}{96}=\frac{7}{18} \frac{c}{A}
$$

Dall'eguaglianza ora stabilita risulta in primo luogo che il valore del rapporto $\frac{\lambda \alpha}{c^{2}}$ 
già considerato nell'Introduzione ${ }^{\mathrm{ro}}$ ), relativo a un intervallo di tempo a un istante del quale la velocità di dilatazione dell'elettrone si annulli, non puó risultare inferiore a $\frac{7}{9}$. Infatti all'istante in cui $\psi$ si annulla il valore della grandezza dell'accelerazione pei punti della sfera di contorno dell'elettrone, in base alla ( 18 ), risulta

$$
A \ddot{\psi}=\frac{7}{18} \frac{c^{2}}{A^{2}} \text {. }
$$

D'altra parte è evidente che allo stesso istante la lunghezza della massima corda dell'elettrone è data da $2 A$. In conseguenza dovrà essere

$$
\frac{\lambda \alpha}{c^{2}} \leq \frac{A \ddot{\psi} \cdot 2 A}{c^{2}}=\frac{7}{9} .
$$

Con ciò l'asserto resta dimostrato. Al tempo stesso vien messo in evidenza che $\mathrm{i}$ moti dilatatori di un elettrone sferico pei quali ad un istante è $\psi=0$, sono allo stesso istante accelerati. Per le osservazioni finali del $\$ precedente, ciò permette evidentemente di asserire che tutti i movimenti dilatatori di un elettrone sferico pei quali ad un istante $\dot{\psi}$ è poco discosto da zero sono individuati dalla $(I)$ in un intervallo di tempo che necessariamente è limitato nel futuro, e in tale intervallo di tempo sono costantemente accelerati, tanto se all'istante in questione è $\psi>0$, cioè l'elettrone tende ad espandersi, quanto se a tale istante è $\dot{\psi}<0$, cioè l'elettrone tende a restringersi. Di più in quest'ultimo caso si puó anche provare che il movimento dell'elettrone cesserà di essere un movimento di contrazione per divenire un movimento di costante espansione prima della fine dell'intervallo di tempo di validità della $(I)$. Infatti in caso contrario la $(I)$ cesserebbe d'individuare il movimento dell'elettrone a un istante in cui $\dot{q}$ avrebbe un valore (negativo) prossimo a zero; ció che è assurdo perchè, essendo $\left(\frac{\partial^{2} l_{a}}{\partial \dot{q}^{2}}\right)_{\dot{q}=0}>0$, in prossimit dello zero $\frac{\partial l_{a}}{\partial \dot{q}}$ è costantemente crescente.

15. La risoluzione della $(I)$ si eseguisce senza la minima difficoltà, se si ammette che il moto preso in esame sia lento: precisamente se si ammette che per esso nello sviluppo di $l_{a}$ in serie di potenze di $\dot{q}$, si possano trascurare $i$ termini contenenti a fattore una potenza di $\dot{q}$ superiore alla seconda.

In tale ipotesi la $(I)$ si riduce evidentemente alla seguente

che puo anche scriversi

$$
\dot{q}=\dot{q}_{\mathrm{c}}+\frac{7}{\mathrm{I} 8}\left(t-t_{\mathrm{o}}\right) \frac{c}{A}\left\{\mathrm{I}+\frac{9}{7} \dot{q}_{\mathrm{o}}^{2}\right\}
$$

$$
\dot{\psi}=\dot{\psi}_{\mathrm{o}}+\frac{7}{\mathrm{I} 8}\left(t-t_{\mathrm{o}}\right) \frac{c^{2}}{A^{2}}\left\{1+\frac{9}{7} \dot{q}_{\mathrm{o}}^{2}\right\} .
$$

ro) Vedi Introduzione. 
Ne segue immediatamente, poichè per $t=t_{0}$ si assume $\psi=\mathrm{I}$,

$$
\left.\psi=\mathrm{I}+\left(t-t_{0}\right) \dot{\psi}_{0}+\frac{7}{36}\left(t-t_{\mathrm{c}}\right)^{2} \frac{c^{2}}{A^{2}} \mathrm{I}+\frac{9}{7} \frac{A^{2} \dot{\psi}_{0}^{2}}{c^{2}}\right\} .
$$

Questo risultato, come subito si vede, conferma pienamente nel caso speciale dei moti lenti quanto abbiamo provato in generale per i moti dilatatori di un elettrone sferico. Di più pone in evidenza che per tali movimenti il rapporto $\frac{\lambda x}{c^{2}}$ gid precedentemente considerato, non puó risultare inferiore a $\frac{7}{9}$.

Padova, giugno 1913.

ANTONIO SignORINI. 